\title{
Tiempo, evidencialidad y miratividad en guaraní paraguayo y español de contacto: ra'e y había sido*
}

\author{
Tense, evidentiality and mirativity in Paraguayan Guaraní and Contact \\ Spanish: ra'e and había sido
}

\author{
Javier Carol y Alicia Avellana \\ Consejo Nacional de Investigaciones Científicas y Técnicas (CONICET) \\ Universidad Autónoma de Entre Ríos . Universidad de Buenos Aires
}

\begin{abstract}
Resumen. En guaraní paraguayo y otras variedades de esta lengua existe un morfema libre ra'e que presenta valores de evidencial indirecto, mirativo y pasado, aunque con frecuentes contraejemplos en los que prima facie la evidencia aparece como directa, la miratividad no se observa y el valor temporal es de presente. En sus usos evidenciales y mirativos equivale frecuentemente, en el español paraguayo, a la expresión adverbial había sido. El presente artículo se propone situar ra'e y había sido en el contexto de debates recientes sobre tiempo, aspecto, evidencialidad y miratividad, y sugerir una explicación unificada para sus diversos valores a partir de la noción de Tiempo de Adquisición de la Evidencia (Lee 2011). A partir de esto, asimismo, establecemos diferencias entre ambas expresiones y otros evidenciales semejantes en otras lenguas, incluyendo los usos evidenciales de las perífrasis haber + PARTICIPIO documentadas en otras variedades sudamericanas.
\end{abstract}

Palabras clave: guaraní, evidencial, mirativo, perfecto.

Abstract. In Paraguayan Guaraní and other varieties of this language there is a free morpheme ra'e showing values of indirect evidential, mirative and past, though with frequent counterexamples

Data de recepción: 24-06-2017 - Data de aceptación: 29-05-2018.

* La presente investigación fue parcialmente financiada por los proyectos PIP 1122015-0100386 (CONICET, Dir. L. Kornfeld) y UBACyT 20020160100106BA (Universidad de Buenos Aires, Dir. C. Messineo). Agradecemos a nuestros informantes y a los evaluadores anónimos de Verba. 
in which prima facie the evidence appears as direct, there is no mirativity and the temporal value is present. In its evidential and mirative uses it often corresponds to the Paraguayan Spanish adverbial expression habia sido. The goals of this paper are to place ra'e and habia sido in the context of recent debates on Tense, Aspect, Evidentiality and Mirativity, and to propose a unified explanation for their distinct values making use of the notion of Evidence Acquisition Time (Lee 2011). We furthermore establish differences between both expressions and similar evidentials in other languages, including the evidential uses of the periphrasis haber + participle documented in other South American varieties of Spanish.

Keywords: Guarani, evidential, mirative, perfect.

\section{INTRODUCCIÓN}

En guaraní paraguayo (gp), correntino, mbyá y otros existe un morfema libre ra'e, ubicado generalmente al final del sintagma verbal (aunque también siguiendo al núcleo predicacional) que ha recibido variadas descripciones que involucran tiempo, evidencialidad y miratividad:

(1) Descripciones de ra'e:

a. Tiempo pasado: "perfecto o pluscuamperfecto en preguntas o con relación a algo sobreentendido" (Guasch 1956: 125); "pasado próximo" en interrogaciones y subordinadas atácticas (Melià et al. 1958: 69, 139); “past” (Gregores \& Suárez 1967: 144), y otros.

b. Evidencial/mirativo: "conocimiento que adquiere el hablante o su sorpresa al enterarse (...) de la realización de una acción" (Krivoshein \& Acosta 2007: 101); "inferencia del hablante (...) evidencialidad "no atestiguada"” (Dietrich 2010: 71), "non-expected evidential marker" (Tonhauser 2006), y otros.

c. Ambos: "afirmación admirativa de algo pasado" (Melià et al. 1958: 70).

Efectivamente hay indicios que justifican la asociación de $r a^{\prime} e$ con cada una de estas categorías. La miratividad es una categoría relativamente reciente en la teoría lingüística difundida en especial a partir del trabajo de DeLancey, quien la define como un indicador de información nueva para el hablante (DeLancey 1997, 2001); la noción ha sido luego ampliamente discutida y redefinida en la bibliografía, véase $\$ 2.2$ abajo. El valor mirativo de ra'e puede verse en (2); en estos casos, suele coocurrir con la partícula enfática niko (ningo, ngo) o la modal/evidencial nipo (nimbo, mbo) (Melià et al. 1958: 70)'.

A menos que se indique la fuente, los ejemplos provienen del trabajo de los autores con hablantes bilingües, tanto elicitados como ofrecidos espontáneamente o escuchados, y todos ellos chequeados 
(2)
a. ¡Re-ju
nipo/niko
ra'e!
2sg.ACT-venir PART $R A^{\prime} E$
'¡(Así que) has venido/viniste!'

b. Rei-kove nipo/niko ra'e!

2sg.ACT-vivir PART RA'E

‘(Así que todavía) estás vivo!'

(Melià et al. 1958: 70)

En estos casos, ra'e suele equivaler a había sido (que) en español paraguayo (ep), variedad que, como es bien sabido, ha estado en intenso contacto con el guaraní desde la conquista española; cf. (3). Esta expresión se documenta también en zonas del noreste de la Argentina. Había sido (que) es allí una forma invariable y sintácticamente opaca que puede preceder al verbo, seguirlo, o bien seguir a todo el sintagma verbal, y RAE (2009: 1789) la caracteriza como una "expresión adverbial"?. (En adelante, incluiremos esta expresión entre paréntesis en la glosa española cuando corresponda en ep, y solo separaremos el ep del español general en la glosa cuando la diferencia exceda el añadido de había sido).

(3) a. ¡Había sido (que) viniste! / ¡Viniste, había sido!

b. ¡Había sido (que) seguís vivo! / ¡Seguís vivo, había sido!

El valor evidencial y, más concretamente, de evidencial indirecto de ra'e/había sido se observa en el hecho de que (2a) y (3a) solo son felices si el hablante tiene

con dos o más hablantes nativos. Abreviaturas en las glosas: 1, 2, 3 primera, segunda y tercera personas; ACT: activo; ART: artículo; CAUS: causativo; COMP: complementante; CONJ: conjetural, inferencial o futuro; DECL: declarativo; DIM: diminutivo; DUR: durativo; EV: evidencial; FUT: futuro; IMPF: imperfectivo; incl: inclusivo; INCT: inactivo; KURI: marcador de pasado kuri; LOC: nominalizador locativo; MIR: mirativo; MOD: modal; NEG: negación; NOM: nominativo; OBL: oblicuo; PART: partícula; PART.PAS: participio pasado; PAS: pasado; PAS.E: pasado experimentado; PAS.NE: pasado no expermientado; PERF: perfecto; pl/PL: plural; POS: posesivo; PRES: presente; PROG: progresivo; PROSP: prospectivo; sg: singular; $R A^{\prime} E$ : evidencial $r{ }^{\prime}$ 'e; $R A K A{ }^{\prime} E$ : evidencial raka' $e$; REL: relacionante; RELAT: relativo. Los símbolos $>\mathrm{y}<$ indican posterioridad y anterioridad temporal, respectivamente. Sobre la distinción entre clíticos y afijos en guaraní, no hay consenso en la bibliografía; dado que no es central para los propósitos del presente artículo, y que justificar nuestra posición requeriría más espacio del deseable, la ignoramos aquí (véase discusión en Carol 2015: 883-887).

2 Aunque la semántica sea similar, no debe confundirse este uso "adverbial" de había sido, propio del contacto con el guaraní, con usos propiamente predicativos de había sido + adjetivo, que ocurren en el habla rural de vastas zonas de la Argentina, aun donde no ha habido contacto significativo con el guaraní, cf. ¡Cha que habia sido salame! (Kany 1970: 206), y otras zonas de Sudamérica tan alejadas como Colombia (Kany, op. cit.). Véase Avellana (2013: 37-38) para diferencias entre había sido del ep y los usos productivos del pluscuamperfecto con valor evidencial/mirativo. 
exclusivamente evidencia indirecta de la previa venida del oyente - p. ej. su presencia frente a él en el momento de habla- pero no si ha sido testigo del evento de su llegada.

Finalmente, el valor temporal de ra'e se nota en los siguientes casos, donde los ejemplos de (a) contrastan con los de (b) por su interpretación en pasado. Nótese que en estos casos "puramente temporales" el ep no utiliza había sido.
a. ¿Moõ-pa re-ĩ ra'e?
dónde-PART 2sg.ACT-estar RA'E
‘¿Dónde has estado/estuviste/estabas?'
b. ¿Moõ-pa re-1?
dónde-PART 2sg.ACT-estar
¿¿Dónde estás?’

(5)
a. La fiesta oi-mé-ne h-óga-pe ra'e.
la fiesta 3ACT-estar-CONJ 3POS-casa-en $R A$ ' $E$
'La fiesta habrá sido en su casa'. (Conjetura o inferencia)
b. La fiesta oi-mé-ne h-óga-pe hína.
la fiesta 3ACT-estar-CONJ 3POS-casa-en PROG
'La fiesta será en su casa'. (Conjetura o inferencia)

(6)
3ACT-saber si.PAS
3ACT.decir-MOD
2sg.OBL $R A^{\prime} E$
a. Oi-kuaa rire, he'i-mo'ã ndéve ra'e.
'Si lo \{supiera/hubiera sabido\} te lo habría dicho'.
b. Oi-kuaa rire, he'i-mo'ã ndéve.
3ACT-saber si.PAS 3ACT.decir-MOD 2sg.OBL
'Si lo supiera te lo diría'.

De lo expuesto se desprende que una explicación unificada de todos estos valores es una tarea compleja. El cuadro de ra'e se completa con los casos en los que los valores mirativo, evidencial o temporal no parecen obtenerse. Así, en (2b), no hay en apariencia ni evidencia indirecta ni pasado: la eventualidad ocurre en el momento de habla y es atestiguada por el hablante; obsérvese además que el valor de presente se refleja en la equivalencia al ep, frente al pasado imperfecto que se halla normalmente en el español general ([jAh,] estabas vivo!). Tampoco es claro el papel evidencial de $r a$ 'e en preguntas o en (5a), donde la modalidad/evidencialidad parece aportarla -ne mientras que ra'e aparentemente se limita a indicar pasado, interpretándose por debajo de $-n e$. Y en cuanto a los valores mirativos, es discutible que $r a{ }^{\prime} e$ exprese sorpresa en expresiones como (7), donde $r a^{\prime} e$ aparece en una subordinada, ya que allí el hablante no está sorprendido en el momento de habla por la caída del lapacho. Lo 
mismo vale para la contraparte en ep había sido. Tampoco es claro que haya sorpresa en las preguntas con ra'e como (4a), ni en las declarativas con -ne como (5a).

$\begin{array}{lllllll}\text { O-topa } & \text { peteĩ } & \text { aratiri } & \text { o-trosa } & \text { ra'e } & \text { la } & \text { tajy } \\ \text { 3.ACT-encontrar } & \text { DET } & \text { rayo } & \text { 3ACT-romper } & R A^{\prime} E & \text { DET } & \text { lapacho } \\ \text { oi-mé-va } & \text { óga } & \text { r-enondé-pe. } & & & \\ \text { 3.ACT-estar-REL } & \text { casa } & \text { REL-delante-en } & & & \end{array}$

'Encontró que un rayo había partido el lapacho que estaba delante de la casa', ep 'encontró que había sido un rayo partió el lapacho...'.

(Melià et al. 1958: 140)

En relación con la teoría lingüística, la relevancia del estudio de $r a$ 'e reside en la diversidad de categorías que involucra, de modo que su esclarecimiento indudablemente contribuirá a comprender mejor la interrelación entre estas. Además, dado que el guaraní no parece codificar gramaticalmente el tiempo absoluto (cf. Tonhauser 2011) o, al menos, la distinción presente-pasado, es de suponerse que esta interrelación requerirá una explicación a veces diferente de la que se ha propuesto para casos análogos en las lenguas europeas.

Los propósitos del presente trabajo son los siguientes. Primero, describir los contextos de uso de ra'e y había sido; segundo, situar ambas expresiones en el contexto de debates teóricos actuales sobre evidencialidad, miratividad y tiempo; tercero, sentar las bases para un análisis unificado de sus diversos valores; y cuarto, comparar y contrastar ra'e con su equivalente en ep había sido y este último, a su vez, con fenómenos semejantes de otras variedades de español de contacto.

El resto del artículo se organiza de la siguiente manera: la sección 2 expone algunas cuestiones teóricas y empíricas generales sobre miratividad, evidencialidad y tiempo/ aspecto, especialmente en la lingüística formal y en los aspectos que resultan relevantes para el presente artículo; la sección 3 describe en forma sucinta el modo en que se interpretan temporalmente los predicados en guaraní en vista de la ausencia de tiempo gramaticalizado o, al menos, de una oposición presente-pasado; la sección 4 recapitula sobre los valores evidenciales, mirativos y temporales de ra'e y había sido respecto de lo expuesto en la sección 2, presenta aparentes contraejemplos a cada caso y reformula las preguntas planteadas en esta sección 1; la sección 5 presenta una propuesta informal de explicación unificada de ra'e; la sección 6 discute algunas consecuencias de la propuesta, la contrapone a otras y especula sobre cuestiones que quedan sin suficiente explicación; la sección 7 indaga sobre las posibles razones de la "elección" de una forma fosilizada del pluscuamperfecto como equivalente de ra'e y contrasta había sido con los usos evidenciales y mirativos de haber + participio en otras variedades sudamericanas de contacto; finalmente, la sección 8 presenta las conclusiones. 


\subsection{Sobre las variedades de guaraní y español estudiadas}

Se conoce como guaraní paraguayo a la variedad de guaraní hablada principalmente por la población criolla, es decir, que no se autorreconoce como indígena, en contraposición al guaraní "étnico", hablado por diversas etnias indígenas, más allá de que muchos indígenas aprenden el gp como segunda lengua o incluso primera. Este gp se ha extendido, por migración reciente, a las zonas limítrofes de la Argentina, así como a Buenos Aires y otros centros urbanos. Un guaraní "criollo" se habla también en la provincia argentina de Corrientes desde época colonial; algunos autores lo consideran una variedad de gp (Melià 1992, Dietrich 2002), mientras que otros destacan particularidades que lo alejan de este (Cerno 2010). En cuanto al español analizado aquí, se trata de la variedad coloquial, aun cuando algunas de sus características, incluida la expesión había sido, lleguen a algunas publicaciones periodísticas, como puede verse abajo.

La mayoría de la población paraguaya es bilingüe gp-ep: casi un 69\% según Estigarribia (2015: 184) en base a datos del último censo, realizado en 2012. No obstante, en las grandes ciudades una proporción significativa de personas aprenden el gp solo como segunda lengua o incluso no lo aprenden en absoluto, mientras que en ciudades pequeñas o zonas rurales puede verse similar situación con el ep. Además de esto, hay integrantes de comunidades indígenas y menonitas que no hablan ni una ni otra lengua, especialmente en la región occidental del país.

Entre el gp y el ep son frecuentes tanto el cambio de código como los préstamos en cualquier nivel. Esta profusa influencia mutua se conoce como jopara (yopará). El jopara ha sido definido como una variedad de guaraní y, en menor medida, de español, o incluso como una "tercera lengua". Creemos que la caracterización más adecuada de lo que sucede en Paraguay es la de Estigarribia (2015), quien considera al jopara más bien una práctica consistente en cambio de código a gran escala ("[se sugiere] to reserve... the term Jopara for the mixed lect derived from Guaraní-Spanish code switching"; ibid.: 217).

Esto lleva a reconocer que lo que aquí llamamos ep y gp son, en buena medida, abstracciones metodológicas, ya que es dudoso que existan ep y gp "puros", tanto a nivel individual (incluso en monolingües) como a nivel social (ibid.: 184), sino que hay más bien un continuum entre ambos extremos (Gynan 2001, Penner 2007, Estigarribia 2015).

Los calcos funcionales del gp en ep son también abundantes; de hecho, proponemos aquí que había sido es un calco de ra'e (o quizá de niko/nipo ra'e). Pero no solo sucede que había sido calca ra'e: ra'e puede insertarse como préstamo en una 
oración en ep (8), e incluso puede coocurrir con había sido en la misma oración, sea el resto de esta en gp o -más frecuentemente- en ep, (9) (nótese que luego, resignificado aproximadamente como 'por cierto' en ep, es a su vez un calco de voi). Estimamos que esto justifica suficientemente el tratar había sido y ra'e de manera conjunta.

(8) Su amada esposa ra'e tenía otro marido (http://www.diariopopular.com.py, última consulta 6/2012)

(9) a. Había.sido la che dúo iñ-akã r-aku peteĩ morocha había.sido DET 1sg.POS amigo 3POS-cabeza -REL-caliente un morocha porã-re oi-kó-vo ra'e.

bello-por 3ACT-estar-COMP $R A^{\prime} E$

'Había sido que mi amigo se enamoró perdidamente de una bella morena' (Traducción del mismo hablante). (http://www.portalguarani.com, última consulta 6/2012)

b. Y había sido ra'e cuando hacés dieta tu cuerpo debe acostumbrarse a ella para abandonarla o, si no, subís el doble. (http://www.diariopopular.com.py, última consulta 6/2012)

\section{MIRATIVIDAD, EVIDENCIALIDAD Y TIEMPO: UN ESTADO DE LA CUESTIÓN}

\subsection{Evidencialidad}

La evidencialidad suele comprenderse como la categoría que se refiere a la naturaleza de la fuente de información de que dispone el hablante respecto del contenido de la proposición (véase Willett 1988; Aikhenvald 2004, entre otros). Existe una discusión en la bibliografía respecto de si debe incluirse dentro de la modalidad (Kratzer 1991, Izvorski 1997, Matthewson et al. 2007, entre otros) o considerarse una categoría independiente (De Haan 1999, Aikhenvald 2004, entre otros). En lingüística formal, los modales se consideran operadores de nivel oracional que califican la verdad de una proposición (i. e. como posible o necesaria) en mundos posibles, frecuentemente en relación con un trasfondo conversacional implícito (Kratzer 1991). Así, por ejemplo, Juan debe estar en su casa significa que Juan está en su casa es necesariamente verdadera en mundos compatibles con la evidencia de que disponemos (p. ej. la luz de su casa está encendida; modal epistémico), o bien en mundos en que se cumplen las prescripciones (Juan está convaleciente y le han recomendado reposo; modal deóntico), según el trasfondo conversacional. En los estudios formales es usual considerar que al menos algunos evidenciales son modales epistémicos con la particularidad de que 
no codificarían necesidad o posibilidad sino solo la fuente de la información (p. ej. Matthewson et al. 2007).

Con todo, se ha sostenido también que no todos los evidenciales serían modales, sino que algunos de ellos codificarían fuerza ilocucionaria, contenido presupuesto o contenido fuera de cuestión [not at issue content], sin aportar contenido proposicional (Faller 2002; Davis et al. 2007 entre otros; para una discusión, véase Matthewson et al. 2007: $\$ 3.2$ y Matthewson 2011: §4). Al respecto, se han propuesto diversos tests para diagnosticar qué evidenciales serían modales y cuáles operadores ilocucionarios. Matthewson et al. (2007), basándose en Faller (2002), propone cuatro que considera válidos: un evidencial es modal si (i) permite subordinación [embedding], si (ii) puede expresarse consenso o disenso con el contenido del modal (posibilidad, necesidad, etc.) y no solo con el de la proposición preyacente ${ }^{3}$, y si (iii) es infeliz cuando el hablante sabe que el contenido de $p$ es falso o (iv) verdadero. Examinamos estos tests en relación con ra'e y había sido en $\S 4$.

\subsection{Miratividad}

El alcance preciso de la noción de 'miratividad' varía ampliamente de un autor a otro. Así, mientras DeLancey $(1997,2001)$ la vinculó originalmente con la codificación de información nueva para el hablante (véase §1), Aikhenvald (2012), por ejemplo, sostiene que puede referirse a cualquiera de los siguientes conceptos desde la perspectiva de uno u otro participante del acto de habla: a) descubrimiento repentino, b) sorpresa, c) mente no preparada, d) contraexpectativa y e) información nueva. Es fácil notar que el deslinde preciso de estos conceptos no es sencillo ya que a primera vista se solapan bastante entre sí. En lo que sigue relevamos solo algunas de las tantas concepciones de miratividad existentes, especialmente las desarrolladas en lingüística formal.

Parte de la bibliografía asocia implícita o explícitamente el rótulo 'miratividad' con la expresión de la sorpresa, y esta con lo que en español y otras lenguas expresa la entonación exclamativa, cf. Peterson 2013, Rett \& Murray 2013, entre otros. Estas últimas autoras consideran, además, que los mirativos implican un acto ilocucionario complejo: aserción de $p$ y evaluación de $p$ como algo inesperado; es decir, asumen que la miratividad implica fuerza ilocucionaria (y también modalidad, según se verá abajo). De modo relativamente similar, Torres Bustamante $(2012,2013)$ postula que los

Es decir, la proposición que actúa como argumento de un operador modal (von Fintel \& Heim 2011: 3). 
mirativos presuponen que los mundos en que $\neg p$ son “mejores" (aquí, más esperables) que aquellos en que $p \mathrm{y}$, aún así, se afirma que $p$.

Rett \& Murray (2013) también notan que, para que una exclamación sea feliz, el hablante debe haber "descubierto" que $p$ inmediatamente antes del momento de habla, y extienden esta observación a la miratividad: cf. (10), donde la exclamación de sorpresa es relativamente tardía en relación con la percepción de $p$, por lo tanto, infeliz:

(10) [Contexto: John y Sue ven a Bill parar en un auto nuevo].

a. Sue, to John: I thought that Bill was still driving a Nissan.

b. John: I did too.

c. Sue: \#(Wow,) Bill has a new car! (Rett \& Murray 2013: 456, ej [9])

A partir de esto, postulan lo que denominan "restricción de recencia" [recency restriction]. Esta se basa en la noción de 'estado meta' [target state] (Parsons 1990: 235): en relación con el evento de enterarse $x$ de $p$, el estado meta es un efecto temporario en $x$ consistente en el cambio de sus expectativas; si además $p$ es inesperada, el efecto es de sorpresa. La restricción de recencia se satisface, pues, si el efecto de sorpresa dura hasta el acto de enunciación $e_{s}$ :

(11) ReCEnCy RESTRICTION: For an event $e_{s}$ of a speaker $i$ uttering a form with at-issue content $p$, and for the event $e_{l}$ of $i$ learning that $p, e_{s}$ satisfies the recency restriction iff $e_{s} \in \operatorname{TARGET}\left(e_{l}\right) . \quad$ (Rett \& Murray 2013: 465, ej. (31))

Por otro lado, la sorpresa no es parte del contenido proposicional, como se prueba, por ejemplo, por el hecho de que no puede negarse. Así, en respuesta a (10) puede negarse la proposición, pero no la sorpresa del hablante, véase (12). Esto es compatible con la explicación de la miratividad -tal como la consideran estas autoras, que relacionan la sorpresa con la exclamación- como fuerza ilocucionaria.

(12) a. No, Bill no tiene un auto nuevo.

b. \#No, no estás sorprendido de que Bill tenga un auto nuevo.

Más adelante argumentaremos que ra'e y había sido no codifican fuerza ilocucionaria/exclamación, sino que este es un rasgo independiente, usual pero no necesariamente asociado a ellos. Que ra'e y había sido merezcan o no el rótulo de 'mirativo' y que la miratividad involucre o no fuerza ilocucionaria dependen, naturalmente, de cómo se defina la miratividad, sobre lo cual hemos visto que no hay unanimidad. Al respecto, no es nuestro propósito entrar en discusiones de términos, sino mostrar 
cuáles de los valores de ra'e/había sido que podrían considerarse "mirativos" en la bibliografía están codificados en estos morfemas y cuáles no, independientemente del rótulo que se asigne a estos. La fuerza ilocucionara, según veremos, no es uno de ellos.

\subsection{Evidencialidad y miratividad}

Los típicos enunciados "mirativos" abordados por la bibliografía desde el trabajo de DeLancey combinan la exclamación (o equivalente) con marcadores de evidencialidad. En efecto, en muchas lenguas del mundo existen morfemas o palabras que se comportan generalmente como evidenciales indirectos y que, en algunos contextos, indican miratividad; Rett \& Murray (2013) los llaman "evidenciales mirativos" [ mirative evidentials]. Cuando esto último sucede, según estas autoras, el requerimiento de la evidencia indirecta no cuenta: la interpretación mirativa se obtiene incluso si el hablante tiene evidencia directa (véanse más casos en DeLancey 1997, 2001). Ejemplo de esto es (13), donde la interpretación mirativa se obtiene, por ejemplo, si el hablante escucha lo que creía que era un auto y, al llegar, ve que es una moto.

(13) Tsafiki (barbacoa, Ecuador)

Moto ju-nu-e.

moto ser-EV-DECL

'Por lo visto es una moto’ o ‘Es una moto!' (Dickinson 2000: 411, apud Rett \& Murray 2013: 458 $)^{4}$

Por otro lado, Peterson (2010) sostiene que la miratividad, que comprende como 'sorpresa', puede codificarse separadamente de la evidencialidad. En el siguiente ejemplo del checheno, según este autor, el perfecto aporta la evidencialidad mientras que $-q$ marca específicamente la miratividad; en palabras de Peterson, $-q$ expresa una miratividad no parasítica, mientras que la de (13) es parasítica ya que se manifiesta mediante un evidencial.
a. Zaara
j-iena.
Zaara j-venir.PERF
'Zaara ha venido'.
b. Zaara j-iena-q.
Zaara j-venir.PERF-MIR
'¡Zaara ha venido!' [El hablante no lo esperaba] (Molochieva 2007, apud Peterson 2010: 141)

La glosa original utiliza apparently, que hemos traducido por 'por lo visto' y no por 'aparentemente', que recibe frecuentemente una lectura focal por la cual se interpreta $p$ como falsa ( $p$ es solo aparentemente verdadera, pero en realidad no lo es). Lo mismo vale para (17). 


\subsection{Tiempo y aspecto}

Las teorías tridimensionales sobre el tiempo basadas en Reichenbach (1947) proponen que las distinciones temporales (y aspectuales) de lenguas como el inglés, el francés o el español pueden explicarse a partir de tres tiempos: el de habla $(\mathrm{H})$, el del evento (E) y un tiempo de referencia (R). Así, el pretérito perfecto simple se explica como $\mathrm{R}, \mathrm{E}<\mathrm{H}$, mientras que el perfecto compuesto como $\mathrm{E}<\mathrm{H}$, R. La reformulación de Klein $(1992,1994)$ redefine R como "tiempo tópico" (TT), es decir, el tiempo del que se habla; H y E y son renombrados como "tiempo de la enunciación [utterance]" y "tiempo de la situación" respectivamente. En lo que sigue, adoptamos en lo general la propuesta de Klein con leves modificaciones terminológicas: utilizaremos "tiempo de habla" (TH) por "tiempo de la enunciación" y "tiempo de la eventualidad" (TEv) por "tiempo de la situación". La de Klein, como otras propuestas actuales, considera estos tres tiempos como intervalos, no como puntos. Esto permite, entre otras cosas, explicar la oposición aspectual perfectivo-imperfectivo; así, en un tiempo perfectivo, al menos el final de TEv está incluido en TT, mientras que en uno imperfectivo TEv incluye a TT. Los tiempos que se orientan desde TH son absolutos o deícticos, mientras que los que se orientan desde TT son relativos. Klein (1992: 537) equipara la relación entre TEv y TT a la noción de aspecto.

Una explicación alternativa a los tiempos perfectos, correspondientes en español al pretérito perfecto compuesto, al pluscuamperfecto y al futuro perfecto, es la desarrollada, entre otros, en Iatridou et al. (2003); cf. otras referencias allí citadas. Allí se propone que los perfectos implican la existencia de un lapso temporal [perfect time span] cuyo límite izquierdo está determinado por adjuntos temporales o contextualmente y cuyo límite derecho, que equiparan grosso modo a R, lo está por el tiempo verbal. En (15a), el límite izquierdo se desprende del adjunto toda la última década ${ }^{5}$, mientras que en (15b) es contextual y podría corresponder, por ejemplo, al comienzo de la vida de Juan en una lectura experiencial del perfecto. El límite derecho está determinado por el tiempo absoluto: en el pretérito perfecto corresponde a un punto coincidente con $\mathrm{TH}$, mientras que en el pluscuamperfecto a uno anterior a $\mathrm{TH}$ y en el futuro perfecto a uno posterior. Esta relación entre el lapso y TH es reflejo del tiempo verbal del auxiliar: presente, imperfecto y futuro, respectivamente; nótese que la

Es cierto, como nota un evaluador anónimo, que cuando el adjunto expresa un punto temporal, $\mathrm{p}$. ej. desde 2001, en español aparece más frecuentemente el presente, en contraste con el inglés: vive en Roma desde 2001 vs. has lived in Rome since 2001. Más allá de esto, sigue siendo válida para el español la afirmación de que el límite izquierdo del lapso es definida por adjuntos o el contexto. 
denominación inglesa - present perfect, past perfect y future perfect respectivamentees más transparente respecto de esta relación.

(15) a. Juan ha vivido en Roma toda la última década.

b. Juan ha visto Nápoles.

En (15a), la lectura más natural es la que se denomina universal: $p$ es verdadera para todo subintervalo perteneciente al lapso temporal del perfecto, mientras que en (15b) solo se admite la lectura existencial: existe al menos un subintervalo perteneciente al lapso temporal del perfecto en que $p$ es verdadera (esto es, Juan estuvo viviendo en Roma todo el lapso en (15a), pero no viendo Nápoles todo el lapso en (15b)). Las interpretaciones universales requieren una eventualidad no delimitada [unbounded]: la eventualidad debe estar en curso durante cierto intervalo (aquí coincidente con el lapso del perfecto), sin haber llegado a un punto final, sea la culminación de una eventualidad télica o la terminación de una atélica (Iatridou et al. 2003: 155). Véase (16), donde las ' $x$ ' representan el intervalo de TEv:

(16) a. Perfecto universal límite derecho del lapso (=R)

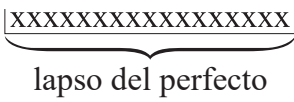

b. Perfecto existencial



\subsection{Evidencialidad, miratividad y tiempo/aspecto}

La interacción entre estas categorías ha sido ya documentada en otras lenguas. Respecto de la relación entre tiempo y evidencialidad, un caso bien conocido es lo que Izvorski (1997) ha llamado "perfecto de evidencialidad" [perfect of evidentiality]: en algunas lenguas, las formas equivalentes al perfecto compuesto del español, o derivadas de ellas, expresan también evidencialidad indirecta (a los usos mirativos de algunas de estas formas ya nos referimos en $§ 2.3$; en realidad, el valor evidencial solo se obtiene en las formas equivalentes al pretérito perfecto del español o al present perfect del 
inglés, pero no en las equivalentes al pluscuamperfecto y futuro perfecto, cf. Izvorski 1997: 223 $)^{6}$ :

(17)
a. Gel-miş-im.
(turco)
venir-PERF-1sg
$\begin{array}{ll}\text { b. } \mathrm{Az} & \text { sam } \\ \text { yo } & \text { ser.1sg.PRES }\end{array}$
dosal.
(búlgaro)
c. Jeg har
venir.PART.PAS
yo haber.1sg.PRES venir.PART.PAS
(noruego)
'He venido (pretérito perfecto)' $\mathrm{y} / \mathrm{o}$
'Por lo visto vine' (perfecto de evidencialidad)

(Izvorski 1997: 222)

La explicación que propone Izvorski para la conexión entre el perfecto y la evidencialidad indirecta se inspira en Iatridou $(2000)^{7}$ para la conexión entre el pasado y la contrafactualidad (cf. la morfología de pasado en Si supiera/hubiera sabido). Esta explicación parte de la noción de exclusión: así como el pasado absoluto expresa que TT -es decir, el dominio contextual de cuantificación temporal- está excluido de TH, esto tiene su correlato en el dominio modal: en las contrafácticas, el dominio contextual de cuantificación de mundos [world quantification], o 'esfera tópica' [Topic Sphere], está excluido del conjunto de mundos epistémicamente accesibles desde el mundo del hablante, es decir, del conjunto de mundos que se sabe constituyen el mundo presente (Izvorski 1997: 234-235; cf. Iatridou 2000). Volviendo al perfecto, este indica que TEv es anterior a TT ( $=\mathrm{R}$ en la representación de [14]) y que, por lo tanto, está excluido de este último; además, si se trata del pretérito perfecto o present perfect, TT coincide con TH; en síntesis, el pretérito perfecto implica que TEv está excluido de TH. Pues bien, el correlato de esto en el dominio modal, atendiendo a la denotación del perfecto de evidencialidad, es que el conjunto de mundos donde se conoce $p$ (tal que $p$ resulta de asignarle especificación temporal a la eventualidad) está excluido de la esfera tópica (correlato de TT), y por lo tanto también del conjunto de mundos accesibles desde el mundo del hablante (correlato de TH). En síntesis, el hablante no tiene evidencia directa de $p$.

En cuanto a la relación entre tiempo y evidencialidad/miratividad, una noción de la que nos serviremos en el presente trabajo es la de 'Tiempo de Adquisición de la Evidencia' (TAE) [Evidence Acquisition Time], desarrollada en el estudio del coreano

\footnotetext{
6 Según Izvorski, en turco la expresión del ejemplo solo expresa hoy perfecto de evidencialidad y no perfecto "normal", aunque deriva históricamente de este último. En cambio, las expresiones del búlgaro y del noruego son ambiguas entre ambas lecturas.

7 En realidad, se cita un manuscrito homónimo previo.
} 
(Lee 2011). El TAE es el tiempo en que el hablante adquiere la evidencia relevante para $p$ dentro del alcance del evidencial (Lee 2011; Smirnova 2011; cf. Koev (2011); cf. también Fleck (2007) sobre una noción similar).

Otro caso en el que también interactúan la miratividad, la evidencialidad y el tiempo, y que resulta aún más cercano al gp/ep, se documenta en variedades de quechua y español andino. En quechua existe un sufijo -sqa que, según resume Faller (2004), ha sido rotulado como "pasado reportativo" (Cusihuamán 1976), "pasado no experimentado" (Cerrón Palomino 1994) y "tiempo de descubrimiento repentino" [sudden discovery tense] (Adelaar 1977; Lefebvre \& Muysken 1988). Este sufijo se contrapone a -rqa, que usualmente indica un pasado atestiguado por el hablante, cf. (18a-b) (aunque sobre otro análisis para ambos sufijos, cf. Faller 2002: 31-32; 2004)

(18) a. Para-sha-sqa. llover-DUR-PAS.NE 'Estaba/estuvo lloviendo'. (El hablante lo infiere al ver el agua en la calle)

b. Para-sha-rqa-n. llover-DUR-PAS.E-3 'Estaba/estuvo lloviendo'. (El hablante ha visto la lluvia caer) (Faller 2004: 46)

En el español de contacto con quechua de Perú, Bolivia y NO de Argentina, -sqa encuentra su correlato en el pluscuamperfecto, que también adopta valores evidenciales y mirativos (véase Kany 1970; de Granda 1994; Faller 2004; Sánchez 2004; Torres Bustamante 2012, 2013, entre muchos otros):

(19) a. Español de Bolivia:

¡Ah! llokalla ['muchacho’], habías venido. (Kany 1970:297)

b. Español del NO argentino:

¿Qué muchos niñitos y tan lindos que había teníu, señora! (Vidal de Battini 1980 (I): 213)

Para explicar estos valores, Torres Bustamante $(2012,2013)$ postula un operador mirativo $M$ que toma una base modal consistente en el conjunto de creencias del hablante; esta base toma, a su vez, un argumento temporal con valor de [pasado], de modo que se obtiene un conjunto de creencias en pasado. Un punto crucial de la propuesta es que, a fin de que $M$ tome [pasado] como argumento temporal, [pasado] no debe interpretarse en ST (o SFlex), que es el dominio de la aserción, sino en SComp, que es el dominio que se atribuye a la miratividad (véase síntesis en Torres Bustamante 2013: 12-13). En 
otras palabras, el pluscuamperfecto de contacto con quechua introduce, según esta autora, un tiempo que se interpreta por fuera de $p$.

\section{LA INTERPRETACIÓN TEMPORAL EN GUARANÍ}

Antes de avanzar con la discusión sobre $r a$ 'e resulta indispensable presentar algunas particularidades del gp en relación con el tiempo, ya que esta lengua difiere en buena medida de las lenguas europeas en base a las cuales se elaboró la mayoría de los conceptos revisados en la sección anterior.

El guaraní no gramaticaliza el tiempo o, por lo menos, la oposición presente/ pasado como lo hacen las lenguas europeas bien conocidas. Un verbo que solo contenga marcas de persona y número pero no de TAM puede interpretarse en presente o en pasado y, en limitadísimos casos, en futuro, y esto depende del contexto y de adjuntos temporales (véase Tonhauser 2011). Esto se ejemplifica en (20), donde los adverbios de tiempo restringen la interpretación temporal del predicado.

(20) a. Kuehe a-jahu.

ayer 1sg.ACT-bañarse

'Ayer me bañé'.

b. Ko'ãga a-jahu.

ahora 1sg.ACT-bañarse

'Ahora me baño'.

c. *Ko'ẽro a-jahu.

mañana 1sg.ACT-bañarse

Significado buscado: 'Mañana voy a bañarme'. (Tonhauser 2011: 260)

En (20c) el verbo sin marca de TAM es incompatible con una interpretación en futuro; esto último requiere morfología extra en el verbo como, por ejemplo, el prospectivo -ta: a-jahú-ta 'voy a bañarme'. Los casos en que el verbo sin marca puede interpretarse en futuro son limitadísimos: Tonhauser halló solo dos contextos sintácticos que se ilustran en (21) (Tonhauser 2011: 273-274). Cada uno de ellos involucra dos cláusulas en las que ambos predicados se interpretan en futuro, aunque solo el primero porta marca de prospectivo; en (21a) ambas cláusulas están coordinadas, mientras que en (21b) la primera es una subordinada causal.

(21)
a. A-jahú-ta
ha (upéi) a-jupi
kolektívo-pe.
1sg.ACT-bañarse-PROs
y luego
1sg.ACT-subi
colectivo-en
'Voy a bañarme y después a tomar el colectivo.' 


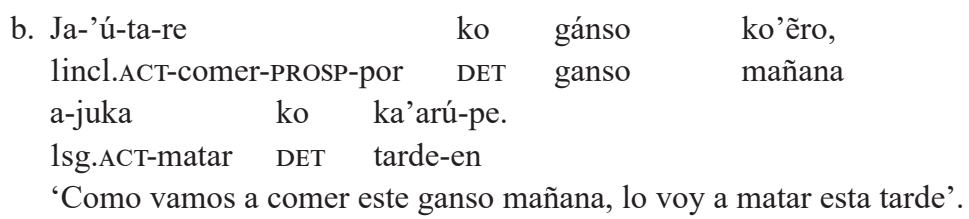

A partir de casos como los de (21) (y de otros argumentos que no pueden discutirse aquí), Tonhauser (2011) considera que el verbo sin marca puede interpretarse no solo en pasado y presente sino también en futuro, y que por lo tanto el guaraní no codifica Tiempo, es decir, es una lengua sin Tiempo.

Un análisis alternativo es suponer que, en vista de la extrema rareza de los casos como los de (21) -y sin mencionar la posibilidad de suponer allí elipsis de la marca de prospectivo- estos pueden dejarse de lado y asumirse que, en realidad, el guaraní sí codifica una distinción temporal, que es entre futuro y no futuro, como muestra (20). Pero aún sobre esto último cabe un reparo: las marcas de futuro del gp no expresan futuro absoluto sino relativo. En efecto, una forma como a-jahú-ta puede significar, dado el contexto adecuado, 'iba a bañarse', lo cual evidencia que -ta no indica posterioridad de TEv o TT respecto de TH, sino de TEv respecto de TT (cf. Tonhauser 2009). Algo similar puede decirse del futuro de obligación vaerã, del desiderativo -se, del inminencial -pota, etc. En conclusión, en cualquiera de los dos análisis el guaraní no gramaticaliza el tiempo absoluto.

Para recapitular, la interpretación temporal de los predicados depende como mínimo de (a) el contexto, (b) adjuntos temporales y (c) marcas gramaticales de TAM (sufijos o clíticos), que incluyen tiempo relativo o aspecto y modalidad, pero no tiempo absoluto. En lo que sigue, asumiremos que tanto (a) como (b) funcionan fijando un TT que, en conjunción con las marcas de TAM (si las hay) determinan la interpretación temporal del predicado. En (20b) el TT fijado por el adjunto incluye o coincide con momento de habla, de allí la interpretación en presente, mientras que el de (20a) lo precede.

Sin embargo, esto no explica todo. En (22a-b) el TT incluye el TH y, con todo, la traducción más probable al ep utiliza el pasado, mientras que en $(22 \mathrm{c})$ recurre al presente:

\footnotetext{
a. Ko_este_día a-hái peteĩ carta. hoy 1sg.ACT-escribir DET carta 'Hoy escribí/he escrito una carta'.

b. Ko_este_día a-jahu. hoy 1sg.ACT-bañarse 'Hoy me bañé/he bañado'.
} 


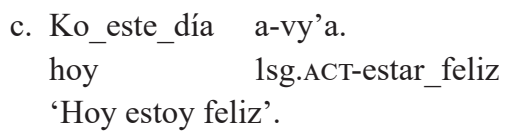

Si bien una explicación de cómo incide el aspecto léxico en la interpretación temporal en gp requiere mucha mayor justificación, asumiremos aquí la hipótesis de que lo anterior se explica debido a que las eventualidades télicas se interpretan por defecto como incluidas dentro del TT, y que lo mismo tiende a suceder con las atélicas dinámicas, i. e. las actividades, salvo cuando reciben una interpretación habitual/genérica, mientras que con los estados y demás actividades ocurre lo inverso: TT está incluido en TEv. Esto último también sucede en presencia del progresivo hína, independientemente del aspecto léxico del predicado. Es decir, las eventualidades télicas son por defecto (i. e. sin ulterior morfología) delimitadas, las actividades tienden a serlo, y los estados son por defecto no delimitados. El punto es que la culminación o terminación de la eventualidad se interpreta como anterior al $\mathrm{TH}$, de allí que las eventualidades delimitadas "prefieran" interpretaciones en pasado y las no delimitadas en presente; véanse argumentos al respecto en Tonhauser (2011) ${ }^{8}$.

Por último, entre las marcas de TAM, hay dos que tienen especial interés para el presente trabajo: kuri y raka'e. Ambas son formas libres, tienen una distribución sintáctica similar a la de ra'e y puede considerarse que integran un paradigma común. Kuri funciona en cierto modo como un correlato de $r a ' e$, en el sentido de que también expresa pasado, pero cuando la evidencia es directa, cf. (23), y aparece frecuentemente en respuestas a preguntas que incluyen $r a^{\prime} e$, cf. (24). Ha sido analizado como indicador de pasado reciente (Krivoshein \& Acosta 2007: 93) del mismo día (Gregores \& Suárez 1967: 154, 231; Melià et al. 1958: 68; Guasch 1956: 125) o de "dos o tres días más antes" (Guasch \& Ortiz 1996: KURI)"
a. O-ho
ra'e.
3ACT-ir
$R A^{\prime} E$
'(Había sido que) se fue'. (Evidencia indirecta)

Aquí debe atenderse también a las características de los adjuntos: ko'ãga denota normalmente un intervalo más breve que ko este día 'hoy', por lo que admite más fácilmente que -jahu 'bañarse' se interprete como incluyendo al TT, como en (20b) y no como incluido en él, como en (22b). En contextos como el de (20b) resulta lo más natural que ocurra el progresivo hína, que fuerza la lectura no delimitada del predicado.

$9 \quad$ Para el guaraní kaiwá, Taylor (1984) glosa kuri como 'agora mesmo’ y lo traduce por la perífrasis en portugués ter + PARTICIPIO PASADO. Dooley (2006) para el mbyá lo explica como "Hoje mais cedo ou mais tarde (ação presenciada pelo falante)", y aporta ejemplos con referencia temporal futura -algo imposible en gp- en las que ocurre la partícula de futuro -rã. En esta variedad puede funcionar incluso como término de preposición: kuri peve 'até hoje'. 
$\begin{array}{ll}\text { b. O-ho } & \text { kuri. } \\ \text { 3ACT-ir } & \text { KURI } \\ \text { 'Se fue/se ha ido'. (Evidencia directa) }\end{array}$

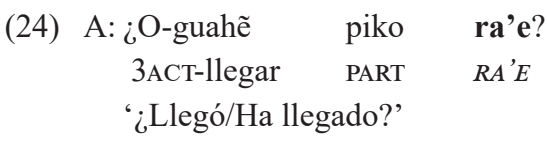

B: N-o-guahẽ-i kuri.

NEG-3ACT-1legar-NEG KURI

'No llegó/No ha llegado'.

Por su parte, raka'e también expresa pasado y evidencia indirecta pero, a diferencia de ra'e indicando pasado remoto o, al menos, más distante de TH que ra'e. Ha recibido descripciones como "pasado remoto" (Krivoshein \& Acosta 2007: 93; Melià et al. 1958: 69, 139; Gregores \& Suárez 1967: 44), "perfecto o pluscuamperfecto en preguntas o con relación a algo sobreentendido" (Guasch 1956: 125), "pasado" (Guasch \& Ortiz 1996: RAKA'E) y similares; para el mbyá, Dooley (2006: [A]RAKA'E) da "ação no tempo remoto, geralmente no passado e não presenciado pelo falante". Al igual que ra'e, ocurre en preguntas pero, a diferencia de aquel, no admite usos mirativos, ya que supone algún tiempo de procesamiento mental entre la adquisición de evidencia y el presente.

(25) a. ¡O-ho ra'e!

3ACT-ir RA'E

‘(Había sido que) se fue!' (Evidencia indirecta)

b. O-ho raka'e

3ACT-ir RAKA'E

'Se fue'. (Hace tiempo; evidencia indirecta; infeliz como mirativo)

(23) a. ¿Ndé-iko re-ho ra'e escuéla-pe?

2sg-PART 2sg.ACT-ir RA'E escuela-a

¿¿Has ido/fuiste a la escuela?’ (típicamente, hoy)

b. ¿Ndé-iko re-ho raka'e escuéla-pe?

2sg-PART 2sg.ACT-ir RAKA'E escuela-a

¿¿Has ido/fuiste a la escuela?’, i. e. ‘¿Estás escolarizado?’

Taylor (1984) propone para el kaiwá el siguiente sistema de oposiciones temporales/evidenciales, el cual, al menos como primera aproximación descriptiva, luce 
válido también para el gp (araka'e = gp raka'e; va'ekwe = gp va'ekue; la traducción de los parametros desde el portugués es nuestra)

CUADRo 1: temporales/evidenciales en kaiwá según Taylor (1984)

\begin{tabular}{|l|l|l|l|}
\hline & & \multicolumn{2}{|c|}{ grado de presencialidad } \\
\hline \multirow{2}{*}{ pasado } & & directa & indirecta \\
\hline & próximo & kuri & ra'e \\
\cline { 2 - 4 } & remoto & va'ekwe & araka'e \\
\hline
\end{tabular}

\section{RA'E Y HABÍA SIDO EN RELACIÓN CON DISCUSIONES TEÓRICAS SOBRE EVIDENCIALIDAD, MIRATIVIDAD Y TIEMPO/ASPECTO}

En esta sección precisamos la caracterización de ra'e y había sido a la luz de las nociones presentadas en $\$ 2$ y refinamos las preguntas que guían el artículo, para las cuales se ofrece una respuesta en $\S 5$.

\subsection{Evidencialidad}

En relación con la evidencialidad, ra'e y habia sido aparecen en principio como evidenciales indirectos, en tanto los enunciados que integran solo son felices si el hablante tiene una evidencia indirecta de la eventualidad pero no si ha sido testigo de ella, como en (2a-3a), aunque con importantes excepciones cuando el predicado es no delimitado, como en (2b-3b) (ejemplos repetidos abajo); esto se discute en §5.2.

(2)
a. ¡Re-ju
nipo/niko ra'e!
2sg.ACT-venir PART RA'E
' (Así que) has venido/viniste!'
b. Rei-kove nipo/niko ra'e!
2sg.ACT-vivir PART RA'E

‘(Así que todavía) estás vivo!’ (Melià et al. 1958: 70)

(3) a. ¡Había sido (que) viniste! / ¡Viniste, había sido!

b. ¡Había sido (que) seguís vivo! / ¡Seguís vivo, había sido!

De cualquier modo, lo indisputable es que ra'e y había sido codifican evidencia percibida por los sentidos -típicamente de un resultado o consecuencia de la 
eventualidad, aunque puede ser un reporte también-, sin importar la naturaleza directa o indirecta de la evidencia, y en esto $r a^{\prime} e$ se distingue de otros evidenciales del gp como ndaje/-je, que codifica específicamente evidencia proveniente de reporte, y de -ne, que codifica evidencia obtenida tanto por razonamiento como por percepción de resultados o consecuencias, cf. (5b).

En cuanto al carácter modal de ra'e y había sido, una discusión rigurosa excede largamente los propósitos del presente trabajo, por lo que nos limitaremos a consignar que pasan, por lo menos, los tests de modalidad (i-iii) mencionados en §2.1. El primero de ellos se basa en la idea de que los operadores ilocucionarios no pueden subordinarse; al respecto, (7) muestra que ra'e y había sido pueden aparecer en subordinadas, además de que ra'e también admite subordinación semántica, según muestra su ocurrencia en preguntas como (4a) (sobre esta prueba, véase Rivero 2014); ambos ejemplos se repiten abajo.

$\begin{array}{lllllll}\text { O-topa } & \text { peteĩ } & \text { aratiri } & \text { o-trosa } & \text { ra'e } & \text { la tajy } \\ \text { 3.ACT-encontrar } & \text { DET } & \text { rayo } & \text { 3ACT-romper } & R A A^{\prime} E & \text { DET lapacho } \\ \text { oi-mé-va } & \text { óga } & \text { r-enondé-pe. } & & & \\ \text { 3.ACT-estar-REL } & \text { casa } & \text { REL-delante-en } & & & \end{array}$

'Encontró que un rayo había partido el lapacho que estaba delante de la casa', ep ‘encontró que había sido un rayo partió el lapacho...' (Melià et al. 1958: 140)

(4)
a. ¿Moõ-pa re-ĩ ra'e?
dónde-PART 2sg.ACT-estar $R A^{\prime} E$
¿¿Dónde has estado/estuviste/estabas?'

El test (ii) parte de que, si un significado relevante puede cuestionarse, rechazarse o ponerse en duda, entonces forma parte del contenido de $p$, como se predice para los modales y a diferencia de los operadores ilocucionarios (véase Faller (2002) por detalles sobre este test). Al respecto, (26) muestra que el contenido modal de ra'e/había sido, que interpretamos como " $p$ es verdadera en vista de la evidencia disponible" puede negarse, sin que ello implique negar $p$ : en este caso, se niega que la luz prendida sea evidencia de que está en su casa sin que ello implique negar que está en su casa. El test (iii) asume que, si ra'e y habia sido afirman que $p$ en vista de la evidencia diponible, entonces $p$ ra'e/había sido será necesariamente infeliz si el hablante sabe que $\neg p$; esto se confirma en $(27)^{10}$.

10 Hemos documentado algún caso en que el hablante sabe que $\neg p$, pero en un contexto a su vez modal (cf. nota 11): 
(26)
A: Juan $\quad$ o-ĩ
h-ógape
ra'e. H-endy
i-lu.
Juan 3ACT-estar 3POS-casa-en $R A$ 'E 3 INCT-estar_encendido 3POS-luz
'(Había sido que) Juan está en su casa. La luz está prendida'.
B: Nahániri, o-heja-nte voi lu h-endy
No 3ACT-dejar-nomás ciertamente luz 3ACT-estar_encendido
o-sẽ vove.
3ACT-salir cuando

'No, siempre deja la luz prendida al salir'. (Cf. Matthewson et al. 2007, ejs. [52-53]).

(27)

$\begin{array}{lllrrrr}\text { a. Juan o-ĩ } & \text { h-óga-pe } & \text { ra'e, } & \text { \#che } & \text { a-hecha } & \text { kuri } \\ \text { Juan } & \text { 3ACT-estar } & \text { 3POS-casa-en } & R A^{\prime} E & \text { yo } & \text { 1sg.ACT-ver } & \text { KURI } \\ \text { o-sẽ } & \text { ha-gue. } & & & & \\ \text { 3ACT-salir } & \text { COMP-PAS } & & & & \end{array}$

Significado buscado: '(Había sido que) Juan está en su casa, pero yo vi que se fue'. (Comentario del informante: "se afirma que está, pero el hablante está confundido". Es decir, solo es aceptable suponiendo confusión del hablante y sin negar la afirmación de que está en su casa).

b. Juan o-ĩ h-óga-pe ra'e, \#péro che ai-kuaa japu-ha. Juan 3ACT-estar 3POS-casa-en $R A^{\prime} E$ pero yo 1sg.ACT-saber mentira-COMP '(Había sido que) Juan está en su casa, pero yo sé que es mentira'.

En cambio, el test (iv) es más problemático; este se basa en que, si el hablante está seguro de que $p$, modalizar $p$ violaría la máxima de cantidad de Grice. Las expresiones p ra'e/había sido pasan este test cuando la evidencia es indirecta; así, (2a-3a) no son felices si el hablante ve venir al interlocutor. Sin embargo, parecen no pasarlo en casos como (2b-3b), donde la evidencia aparece como directa; sobre esto, argumentaremos en §5.2.1 que aún allí hay evidencia indirecta y que, en realidad, el hablante no sabía que $p$ durante el intervalo evaluado por ra'e/había sido.

Debe notarse que la propia Matthewson (2011) descarta posteriormente los diagnósticos (iii-iv) en base a argumentos que no podemos detallar aquí sin desviarnos de la cuestión. Con todo, admite que (iii) se mantiene como válido para algunos evidenciales, concretamente aquellos que se basan en un "trasfondo conversacional realista" [realistic conversational background], y que admiten paráfrasis como En vista de la evidencia disponible... (Kratzer 2012); en estos casos, no se admite una continuación

(i) Ai-mo’ã lú-ma la h-endý-va ra'e!

1sg.ACT-creer luz-ya DET 3INCT-encenderse-REL ra'e

‘Creí que era la luz (había sido) lo que se prendió!' (Escuchado. Contexto: se ha cortado la luz y el hablante, al ver una linterna, cree que ha vuelto la energía. El hablante ya sabe que no es cierto cuando produce el enunciado). 
como 'pero yo sé que es mentira'. En cambio, este no es el caso en un "trasfondo conversacional informacional" [informational], donde una posible paráfrasis es Según el contenido de (Xfuente)..., ya que allí no se compromete la creencia o conocimiento del hablante. Como hemos visto, ra'e y había sido se comportan normalmente como evidenciales de trasfondo conversacional realista (si bien cf. nota 10).

Una cuestión diferente es si $p$ ra'e/habia sido es feliz cuando el hablante no cree que $p$ (cf. Dolzani 2016). El caso de que tales enunciados sean felices no solo es compatible con un trasfondo conversacional informacional, sino también con un análisis existencial del modal: $p$ ra'e/habia sido es feliz en al menos un mundo compatible con la evidencia, no en todos, y ese mundo puede no ser compatible con las creencias del hablante, cf. El mayordomo \{puede/\#debe\} ser el asesino, pero yo creo que es inocente. Efectivamente, el hecho de que expresiones como (28a) sean aceptables para varios hablantes pareciera indicar que tales valores modales existenciales son posibles con $\mathrm{ra}^{\prime}$ (sorprendentemente, la contraparte en ep nos fue rechazada). En cambio, la infelicidad de la continuación en (28b) sugiere lo contrario.

a. Juan ho-'a ra'e, péro che nd-aroviá-i.

Juan 3ACT-caerse $R A^{\prime} E$ pero yo NEG-creer-NEG

'Juan se cayó (había sido), (\#) pero yo no lo creo'. (Basado en Dolzani 2016, ej. [21a])

b. Juan ho-'a ra'e, \#péro i-katu nd-o-’á-i.

Juan 3ACT-caerse RA'E pero 3INCT-posible NEG-3ACT-caerse-NEG

Buscado: 'Juan se cayó (había sido), pero puede ser que no se haya caído'. (Comentario del informante: hay contradicción)

Sea como fuere, los casos de "lectura existencial" de ra'e son más bien raros y no aceptados por todos los hablantes consultados, por lo que los consideraremos marginales y nos centraremos en los casos usuales en que el hablante se compromete con lo afirmado" .

11 Cabe preguntarse si la felicidad de (28a) no podría explicarse asignándole una interpretación en que el hablante se compromete en realidad con $p$, solo que manifiesta su desconcierto frente a ello (i. e. pero yo me resisto a creerlo).

Por otra parte, debe tenerse en cuenta que los evidenciales que no codifican fuerza cuantificacional (i. e. universal o existencial) no son en absoluto una rareza. Según Matthewson et al. (2007), los modales codifican la fuerza o bien la fuente de la evidencia y este último, afirman, es el caso de los evidenciales del st'at'imcets. Según ellos, esta lengua tiene tres evidenciales: reportativo, inferencial y lo que denominan "de evidencia percibida" [perceived evidence]. Ra'e se asemeja al último, con los reparos presentados sobre las interpretaciones existenciales; sobre él, afirman incluso que tiene preferencia por interpretaciones univerales. Los otros dos muestran notables 


\subsection{Miratividad}

En cuanto a la miratividad, ra'e y había sido se comportan en general como un evidencial mirativo del tipo descripto en §2.3. Como podría esperarse, cuando expresan sorpresa esta no forma parte del contenido proposicional; así, por ejemplo, (29a) puede responderse con $(29 b)$, pero no con $\left(29 b^{\prime}\right)^{12}$.



Por otro lado, si por miratividad se entiende sorpresa, la que expresan ra'e y habia sido es "parasítica" de la evidencialidad en los términos de Peterson (2010) vistos en $\S 2.3$, según se desprende de la existencia de usos evidenciales de ra'e y había sido en que no hay sorpresa. Ejemplo de esto es otra vez (7): allí ra'e y había sido no expresan verdadera sorpresa, ya que la eventualidad descripta en la subordinada no es información nueva en el momento de habla, con lo que no satisface la restricción de recencia presentada en $\S 2.2$. Esto es esperable tratándose de una subordinada: si la sorpresa se manifiesta con la ilocución exclamativa, y si la subordinada no admite exclamación, no puede haber allí sorpresa. Y si la miratividad es sorpresa, no puede llamarse miratividad a lo que hay en (7); volveremos sobre esto al final de la sección.

Otra característica de ra'e y había sido común a los demás "evidenciales mirativos" es que eliminan (aparentemente, según argumentaremos) el requerimiento de evidencia indirecta en sus "usos mirativos" (i. e. cuando expresan sorpresa), cf. (2b-3b). En cambio, una característica que no ha sido explicitada para los evidenciales mirativos por Rett \& Murray es que incluso cuando no expresan sorpresa, expresan al menos un descubrimiento, es decir, un conocimiento súbito de la evidencia relevante para $p$, como muestran (7) y (30). En efecto, en (7) el hablante descubre que el rayo

semejanzas con el reporativo -je/ndaje y el conjetural/inferencial (también usado como futuro incierto) -ne, respectivamente.

12 Los intentos de elicitar equivalentes de 'No estás sorprendido de que viniera' no tuvieron éxito; la expresión del texto implica aquella. 
ha roto el árbol en el tiempo tópico, es decir, el indicado por el verbo principal atopa 'encontré'. Es cierto que allí puede argumentarse que el descubrimiento está también implicado por el verbo principal, pero esto no ocurre en (30), que resulta un ejemplo más ilustrativo al respecto: (30) solo es feliz si el hablante "descubre" que el partido había terminado luego de salir de su trabajo, pero no si lo sabía de antes; un contexto posible para (30) es que el hablante se enteró de la finalización al preguntarle a alguien en la calle. Y también hay descubrimiento sin sorpresa en (31), donde, a diferencia de (7), no hay una oración principal cuyo verbo establece el tiempo tópico de la subordinada, sino dos independientes tales que la primera establece el tiempo tópico de la segunda, yuxtapuesta a aquella.

(30) A-sẽ-rõ-guare che-mba'apo-há-gui, 1sg.ACT-salir-cuando-PAS la partido o-pá-ma ra'e. DET partido 3ACT-terminar-ya $R A^{\prime} E$

'Cuando salí del trabajo el partido ya había terminado', ep ‘...había sido que el partido ya terminó' (Ejemplo ofrecido espontáneamente).

(31)

A-topa chupe, o-truquea hína ra'e.

1sg.ACT-encontrar 3:OBL 3ACT-jugar_al_truco PROG $R A^{\prime} E$

'Lo encontré, (había sido que) estaba jugando al truco' (Ejemplo ofrecido espontáneamente).

Esta codificación de "descubrimiento" sugiere fuertemente que ra'e y había sido codifican un TAE como el descripto en $\S 2.5$; la cuestión se retomará en $\S 5$. Esto explicaría también que no puedan usarse como evidenciales narrativos, cf. $(32)^{13}$ :

(32) Ñorairõ guasu o-ñepyrũ mil_noveciento_treinta_y_do-pe ra'e. guerra grande 3ACT-empezar mi_novecientos_treinta_y_dos-en $R A^{\prime} E$ '(Había sido que) la Guerra Grande empezó en 1932'. (Infeliz si se busca un uso narrativo del evidencial).

13 Si acaso ra'e aparece en narraciones, ello se debe a que el narrador ha cambiado la perspectiva y el tiempo de evaluación al aquí-ahora de los hechos narrados, de modo que ra'e expresa un descubrimiento súbito de ese momento. Es decir, algo como lo mostrado en (30)-(31). Cf. el siguiente ejemplo del guaraní kaiwá (Taylor 1984: 20; grafía y glosa nuestras):
(i) Che
renda
o-jere
o-jere.
Ha i-po
1sg.Pos montado 3ACT-dar rodeo
oi-su'u
ra'e
3ACT-dar_rodeo y 3Pos-mano
3ACT-morder
$R A{ }^{\prime} E$
mbói.
serpiente
'Mi caballo se puso a dar vueltas y vueltas. Una cobra lo había picado en la pata delantera'. 
Cuando se relata el origen del mundo, una hazaña de un héroe o una historia cualquiera de la que el hablante no ha sido testigo, normalmente no es relevante cuándo el hablante adquirió conocimiento de lo que narra sino, como máximo, la naturaleza de su fuente, esto es, si es un rumor, una conjetura, un razonamiento basado en resultados, etc. Así, si se trata de un conocimiento de oídas, el hablante de gp probablemente utilizará el reportativo ndaje o -je. En cambio, ra'e y había sido no son felices en tal contex to ya que explicitan un cambio en el estado de conocimiento del hablante que aquí no es buscado. En síntesis, ra'e y había sido siempre expresan un descubrimiento súbito, con o sin sorpresa.

Para concluir la sección, creemos oportuna una recapitulación sobre la terminología que se halla en la bibliografía, ya que frecuentemente las discusiones sobre miratividad se enturbian con cuestiones de términos. Lo que ocurre en (7), (30) y (31) sirve para ilustrarlo. Si uno identifica la miratividad con la información nueva, el descubrimiento o la conciencia súbita, o lo que llamamos aquí "descubrimiento", puede con toda lógica tomar casos como (7), (30) y (31) como evidencia de que la miratividad no implica fuerza ilocucionaria exclamativa, ya que allí hay información nueva, descubrimiento, etc. pero no exclamación; véase p. ej. Olberz (2009). Del otro lado, si se parte de identificar la miratividad con la sorpresa manifestada -al menos en español- por la exclamación, se puede argumentar que en (7), (30) y (31) no hay miratividad sino evidencialidad. Vuélvase a la noción de "miratividad no parasítica" de Peterson (2010), ilustrada por el checheno en (14b): allí un sufijo - $q$ que Peterson llama 'mirativo' codifica la sorpresa, mientras que el perfecto codifica lo que él considera evidencialidad.
b. Zaara j-iena-q.
Zaara j-venir.PERF-MIR
‘Zaara ha venido!' [El hablante no lo esperaba] (Molochieva 2007, apud Peterson 2010: 141)

\begin{abstract}
Ahora bien, el perfecto del español andino, que Olberz considera mirativo, se asemeja claramente al perfecto del checheno, "evidencial" según Peterson, y no al sufijo que este autor llama "mirativo". En síntesis, tanto unos como otros reconocen, implícita o explícitamente, la existencia de dos componentes diferentes -la sorpresa y el descubrimiento- que frecuentemente ocurren juntos pero son independientes, solo que no acuerdan respecto de cuál de ellos debe llamarse mirativo. En lo que sigue, pues, a fin de evitar ambigüedades, al referirnos a la miratividad la especificaremos como sorpresa/exclamación o como descubrimiento.
\end{abstract}




\subsection{Tiempo/aspecto}

En cuanto al tiempo/aspecto, no parece posible explicar $r a^{\prime}$ 'e como un simple pasado, absoluto o relativo, a partir de los tres tiempos de las teorías de Reichenbach y sus derivados ya que, por un lado, hay valores evidenciales que no resultarían suficientemente explicados y, por otro, hay casos en los que ra'e, al igual que había sido, parece admitir una interpretación en presente. Sobre este último punto, considérense dos tipos de casos: (i) las declarativas (incluyendo exclamaciones) en que ra'e no queda bajo el alcance de otro modal y (ii) los demás casos, i. e. declarativas con otros modales y preguntas.

En (i), ra'e, como había sido, normalmente da lugar a una interpretación en pasado, como en (2a) y (3a). Sin embargo, cf. (2b), donde la eventualidad coincide con el momento de habla y la traducción más usual al ep, con había sido, utiliza el presente, (3b); volvemos a repetir los ejemplos abajo.
(2)
a. ¡Re-ju
nipo/niko ra'e!
2sg.ACT-venir PART RA'E
'¡(Así que) has venido/viniste!'
b. Rei-kove nipo/niko ra'e!
2sg.ACT-vivir PART RA'E
‘(Así que todavía) estás vivo!’ (Melià et al. 1958: 70)
(3) a. ¡Había sido (que) viniste! / ¡Viniste, había sido!
b. ¡Había sido (que) seguís vivo! / ¡Seguís vivo, había sido!

Así, (2b) y (3b) aparecen como excepcionales tanto en relación con el tiempo como con la evidencialidad, ya que ra'e y había sido no expresarían allí pasado relativo ni evidencia indirecta. En consecuencia, resulta razonable perseguir una explicación unificada para ambas excepciones. Un examen más detallado de los casos de (aparente) evidencia directa e interpretación en presente muestra que estos se corresponden no solo con estados, sino también con predicados habituales/genéricos y cualquiera que contenga el progresivo hína, como (33). Recuérdese que en $\$ 3$ postulamos que todos estos en gp son por defecto no delimitados; es decir, son no delimitados siempre que no reciban ulterior modificación por morfemas de TAM, adjuntos o información contextual.

\footnotetext{
(33) a. ¡O-mba'apo peteĩ oficína-pe ra'e! 3ACT-trabajar una oficina-en $R A^{\prime} E$ ‘(Había sido que) trabaja en una oficina!'. (Contexto: el hablante acaba de enterarse de dónde trabaja el participante).
} 
b. ¡Re-karu-iti ngo hína ra'e!

2sg.ACT-comer-todavía PART PROG RA'E

¡(Había sido que) todavía estás comiendo! (Contexto: el hablante se acerca a la mesa con intención de retirarle los platos al oyente y se da cuenta de que este no ha terminado).

Nótese, con todo, que la interpretación "presente" no es obligatoria para los predicados no delimitados con ra'e, como (2b). Según lo expuesto en $\S 3$, el gp no gramaticaliza la relación entre el TT y el TH sino que esta es determinada por el contexto, adverbios y marcas de TAM que no involucran el tiempo absoluto. En (2b), TT y TH se solapan, lo cual es el caso por defecto en predicados no delimitados, pero podría construirse una interpretación en pasado si se estableciera contextualmente un TT en el pasado. Así, supóngase que la conversación gira en torno a la ausencia del oyente el martes pasado y, súbitamente, aparece alguna evidencia de que el oyente estuvo allí ese martes. Entonces, una expresión aspectualmente equivalente a la de (2b), como es (34), puede recibir una interpretación traducible al ep por '¡Había sido que estabas ahi (el martes)!', donde el predicado se interpreta en pasado y, además, la evidencia es inconfundiblemente indirecta (i. e. que el oyente esté vivo hoy es evidencia indirecta de que también lo estuvo el martes) $)^{14}$.

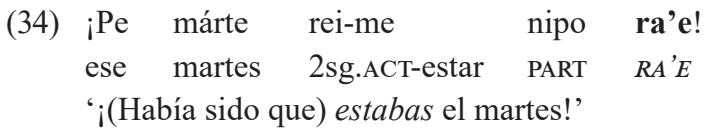

Por otra parte, es incluso posible que un predicado con ra'e (y había sido) exprese que la eventualidad tiene lugar en el futuro, cf. (35); este es el caso cuando ocurren el prospectivo - $t a$, el futuro de obligación vaerã, el desiderativo -se y el inminencial -pota. Allí TEv > TT y, por defecto, TT se solapa con TH, de modo que TEv > TH, igual que si ra'e no estuviera presente, lo que muestra que ra'e se interpreta por encima de estas marcas de TAM. Aquí la evidencia es por fuerza indirecta, en tanto no puede existir evidencia directa de una eventualidad que no ha ocurrido ${ }^{15}$.

14 Hemos sustituido la expresión de (2b) reikove 'estás vivo' por una contextualmente equivalente debido a que aquella resulta artificial en este contexto

15 Otra posibilidad es considerar que este tipo de predicados expresa un estado (lo que es aún más claro en los casos del desiderativo o el futuro de obligación); así, japytáta equivaldría a algo como 'estamos en el estado de haber de quedarnos'. En tal caso, el estado es simultáneo con TH, es decir, se interpreta en presente, y la evidencia es (aparentemente) directa, ya que el hablante en (35) es testigo del estado preparatorio. En este análisis, (35) no es más que una variante de (2b). En cualquier caso, en vista de que en expresiones como estas ra'e y había sido no alteran el tiempo relativo -es 
(35) Ja-pytá-ta (ngo) ra'e.

lincl.ACT-quedarse-PROSP PART RA'E

'(Había sido que) nos vamos a quedar'. (Contexto: dicho por un estudiante que estaba a punto de retirarse del aula por ausencia del profesor y de repente ve llegar a este último. Ejemplo ofrecido espontáneamente).

Respecto de (ii), es decir, las preguntas y las declarativas bajo el alcance de modales, la situación es diferente ${ }^{16}$. Allí la lectura en pasado prevalece, incluso con predicados estativos: cf. (4a), (5a) y (6a), esta última repetida abajo. Recuérdese que en estos casos la equivalencia en gp no incluye había sido.
a. Re-ju rire, a-vy'a -mo'ã ra'e.
2sg-venir si.PAS 1sg.ACT-estar_feliz-MOD RA'E
'Si hubieras venido habría estado feliz'.
b. Re-ju rire, a-vy'a -mo'ã.
2sg-venir si.PAS 1sg-estar_feliz-MOD
'Si hubieras venido estaría feliz'.

Por otra parte, no es claro en (4a), (5a) y (6a) el valor evidencial de ra'e ni que exista un descubrimiento, por lo que, en principio, aquí sí luce razonable un análisis puramente temporal. Se volverá sobre esto en $\S 6.1^{17}$.

\subsection{Sumario}

En fin, un análisis unificado de ra'e, así como de había sido cuando corresponde a $r a$ 'e, debería explicar idealmente (a) que normalmente expresa evidencia indirecta y

decir, la relación entre TEv y TT- de la proposición preyacente, resulta razonable considerarlas casos secundarios.

16 No todos los modales toman alcance sobre $r a^{\prime} e$. De hecho, los radicales parecen quedar por debajo, como es el caso con el futuro de obligación va'erã y el desiderativo -se, cf. el texto. Además, nótese que -mo'ã, que aparece en (6) como lo que consideramos un tipo de frustrativo o conativo (véase Tonhauser 2009; Carol \& Salanova 2018), también funciona como alomorfo del prospectivo - $t a$ en contexto de negación; en tales casos, al igual que con el - $t a$ de (35) y a diferencia del -mo 'ã modal, este -mo 'ã prospectivo tiene alcance por debajo de ra'e: [[Nda-ja-pyta-mo'ã-i] ra'e] '[ ${ }_{\mathrm{RA} \mathrm{E}}$ Había sido que [-мо'

17 Existe un grupo de casos que no consideramos aquí en los que el predicado parece interpretarse en presente y $\mathrm{ra}^{\prime} \mathrm{e}$ toma alcance por encima de los modales. Así, la expresión de (6a), en especial si seguida de ¿ajépa? ‘¿verdad?', puede interpretarse como '(Había sido que) si hubieras venido estaría feliz, ¿verdad?'. En tal caso, ra elhabía sido toma alcance sobre todo el período, y no solo sobre la apódosis. 
pasado y, al mismo tiempo, dar cuenta de los casos de evidencia directa e interpretación en presente; (b) por qué no puede usarse como evidencial narrativo; (c) que expresa sorpresa en unos casos pero no en otros, y (d) por qué no parece codificar evidencialidad en preguntas y bajo modales.

\section{PROPUESTA DE ANÁLISIS}

Nuestra propuesta se basa en que ra'e y había sido introducen un TAE, que definimos como el tiempo en que el hablante adquiere evidencia de $p$. Una expresión de forma $p$ ra'e/habia sido dice que $p$ es verdadera en todo mundo $w$ 'compatible con la evidencia disponible en TAE en el mundo real $w^{18}$. Cuando ocurren ra'e y habia sido, TAE funciona como el tiempo de evaluación, que por defecto coincide con $\mathrm{TH}$, aunque también puede coincidir con (o estar incluido en) TT. El evento de adquisición de evidencia, en tanto cambio de estado mental, es un punto, no un intervalo. Además, en virtud de su coincidencia con (o inclusión en) TH o TT, TAE es un punto definido y siempre identificable, a diferencia de lo que sucede en los evidenciales narrativos. Por otra parte, análogamente al perfecto, ra'e y habia sido implican un lapso previo, cuyo límite derecho es el mismo TAE, durante el cual el hablante no tenía evidencia de $p$. La relación de TAE con TEv, por su parte, no está especificada.

Pese a esta última afirmación, la definición propuesta implica que siempre hay al menos un subintervalo de TEv que no coincide con TAE, según argumentaremos en \$5.2.1, por lo que siempre existe evidencia indirecta. Dado que no resulta necesario hacer referencia al tipo de evidencia en la definición de ra'e/había sido, las "excepciones" al requerimiento de evidencia indirecta mencionadas en $\$ 2.3-i$. e. las que ocurren en sus usos "mirativos", en el sentido de sorpresa- no son un verdadero problema. La anterioridad y la miratividad (sea descubrimiento o sorpresa) tampoco necesitan especificarse en la definición sino que pueden explicarse como valores derivados.

Un punto crucial de la propuesta es que la evidencia está "temporalizada". En esto, nuestro análisis contrasta con el de otros evidenciales que han sido tratados como modales en la bibliografía: las formalizaciones de Izvorski (1997) o Matthewson et al. (2007) hacen referencia a la evidencia disponible en el mundo real (o el mundo del hablante), pero no a ningún tiempo en que se localice tal evidencia. En cambio, ra'e y había sido según la propuesta presentada aquí se asemejan al pluscuamperfecto andino de contacto con quechua según el análisis de Torres Bustamante (2012, 2013),

18 Aunque cf. (28a) y su correspondiente discusión por posibles contraejemplos. 
al perfecto del búlgaro según el de Smirnova (2011) y al coreano -te según Lee (2011), entre otros (véase §2.5).

En lo que sigue, desarrollamos el modo en que la explicación propuesta arriba da cuenta de las preguntas planteadas al final de $\S 4$. Por razones de la argumentación, exponemos primero el análisis de los valores temporales de ra'e/había sido y luego el de los evidenciales y mirativos.

\subsection{Tiempo y aspecto}

A pesar de la analogía que señalamos entre ra'e/había sido y el perfecto, aquellos no codifican realmente un tiempo relativo, es decir, no hacen referencia a la relación entre TEv y TT, sino a TAE. Que no son realmente un tiempo relativo se evidencia en el contraste que manifiestan con la perífrasis de haber + PARTICIPIO del español general en cuanto a la manera en que se combinan con el prospectivo. En español general, el prospectivo ir a + InFINITIVo se ensambla por encima de la perífrasis con haber, un verdadero tiempo relativo, p. ej. va a haber terminado, no por debajo, cf. \#ha ido a terminar. En gp y ep, en cambio, ra'e y habia sido se ensamblan por encima del prospectivo, como muestra (35). Si fueran un verdadero pasado relativo, se esperaría que situaran el TEv con anterioridad al TT que introducen, que aquí se define por el momento en que el hablante percibe que se van a quedar en clase (lo que llamamos TAE), lo cual no ocurre. En cambio, el ensamble de ra'e y había sido por encima del prospectivo no es problemático si se asume que introducen una referencia diferente de TT -TAE-, y es además compatible con un análisis modal/evidencial de ra 'e/había sido, que les asigna un alcance por encima de las marcas de tiempo relativo, según muestran los estudios cartográficos desarrollados a partir de Cinque (1999).

La referencia temporal que codifican ra'e y había sido, entonces, es TAE, que a su vez supone la existencia de un lapso precedente a TAE durante el cual no se tenía evidencia que permitiera afirmar $p$; por el momento, dejamos abierta la cuestión de si este lapso está codificado por ra'elhabía sido o si es solo una implicatura. Por otro lado, ra'e y había sido tampoco ordenan TAE en relación con TEv, como demuestra su mencionada coocurrencia con el prospectivo indicando evidencia de hechos futuros, cf. (35) y, en cuanto a la relación de TAE con TH, esta tampoco es fija: por defecto, TAE coincide con TH, pero también puede situarse en el pasado, cf. (7), (30) y (31).

La figura de (36) expone cómo este esquema temporal da cuenta de expresiones como (2a) y (3a). En TAE, el hablante adquiere evidencia de la eventualidad que ha tenido lugar antes, en el lapso previo durante el cual no tenía tal evidencia, lo cual da cuenta del valor de pasado relativo atribuido a ra'e. El lapso previo precede 
inmediatamente a TAE, que funciona como su límite derecho. Además, en este caso, como sucede por defecto, TAE $=\mathrm{TH}$. Obsérvese que la representación se asemeja a la de un perfecto en la teoría de Reichenbach si se equipara TAE con R, o bien en la propuesta de Iatridou et al. (2003) si se equipara TAE con el límite derecho del lapso del perfecto, véase §2.4; una diferencia, sin embargo, es que lo que llamamos "lapso previo" excluye TAE, como se observa en (36), mientras que en Iatridou et al. (2003) el lapso del perfecto incluye su límite derecho.

(36) ¡Reju nipo ra’e! (=2a)/ ¡Había sido viniste! (=3a)

$$
\text { TAE }(=\mathrm{TH})
$$

TEv: $\mathrm{xxx}$

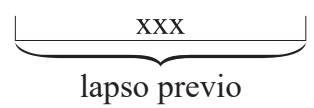

Otro argumento importante a favor de este esquema temporal es que permite dar cuenta de la excepción aparente que representa el "presente" de (2b) (;Reikove nipo/ niko ra'e!) y (3b) ( Habia sido [que] seguís vivo!). En efecto, cabe preguntarse si realmente se interpreta aquí un presente "a secas". Hay elementos que sugieren esto: admite un adjunto como ko 'ãga 'ahora' y su equivalente en ep utiliza normalmente el presente. Sin embargo, esta interpretación no está libre de problemas, según pronto se verá; proponemos por ello asignar aquí a ra'e/había sido una semántica semejante a la de un perfecto universal, es decir, algo como 'has estado vivo (todo este tiempo)', donde un subintervalo del TEv es anterior al TH y otro llega a superponerse a este, véase §2.4. En esta expresión que acabamos de proponer como glosa, el lapso del perfecto está enmarcado por el adjunto todo este tiempo y es, además, equivalente al lapso previo a TAE más TAE $(=\mathrm{TH})$. Es decir: has estado vivo todo este tiempo (incluyendo el presente) durante el cual yo no tenía evidencia de que lo estuvieras.

La representación de (37) grafica cómo sería una interpretación de (2b) en presente puro, de la cual pronto nos apartaremos: allí, la eventualidad está en curso durante $\mathrm{TH}$, como en cualquier presente, y TAE coincide con TH. Lo que indica la flecha plena es lo que el hablante cree en TAE $=\mathrm{TH}$ : ahora tengo evidencia de que estás vivo. ¿Qué sucede con su creencia o estado mental en el lapso previo a TAE? Pues si (2b) es un presente sin más, aquella creencia también debería referirse a esta eventualidad presente, como indica la flecha punteada; en resumen, esta lectura puede parafrasearse por ahora $_{i}$ tengo evidencia de que estás vivo y antes ${ }_{j}$ no tenía evidencia de que (ahora $)_{i}$ ) lo estuvieras. 
(37) Interpretación de (2b) como presente estricto



Sin embargo, no resulta muy razonable suponer que la creencia del hablante en el lapso previo a TAE se refiera a un hecho situado más adelante en el tiempo. Más bien, el hablante tiene, durante dicho lapso, evidencias sobre lo que ocurre en ese momento. En otras palabras: en el lapso previo el hablante carece de evidencias de que su interlocutor esté vivo en ese momento pasado anterior a TAE y, en TAE, pasa a tener evidencia que sí está vivo en ese momento coincidente con TAE y, de ese modo, puede inferir que también lo estaba antes; es decir, la evidencia es sobre distintos momentos, lo cual se grafica en la representación de $(38)^{19}$.

(38) Interpretación propuesta para (2b)

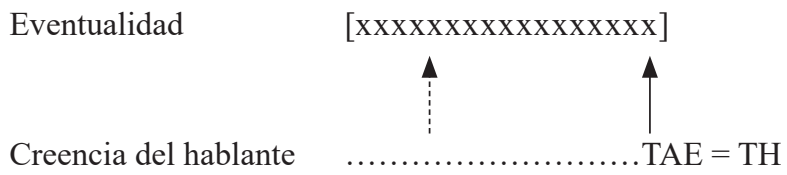

Proponemos considerar que, en (2b), la vieja creencia del hablante, así como la nueva surgida a partir de TAE, se refieren a todo el intervalo previo a TAE más TAE, como en (38) (o bien a distintos subintervalos de este conjunto), y no solo al presente, como en (37). Es decir: ahora tengo evidencia de que durante todo un intervalo (que

19 Obsérvese que el fenómeno que ocurre en (2b) según esta interpretación, presenta semejanzas con la 'lectura de doble acceso' [double accessibility reading] de oraciones como Juan dijo/ha dicho que María está embarazada (cf. Abusch 1997, Giorgi \& Pianesi 1997). Se dice que allí hay doble acceso porque no solo se dice que María está embarazada en el presente de la enunciación, como indica el tiempo verbal de la subordinada, sino que necesariamente también lo estaba en el momento en que Juan lo dijo, de modo que la interpretación temporal del predicado subordinado tiene dos accesos posibles: desde el TH y desde el TEv de la principal. Así como tiene poco sentido suponer que Juan hizo una predicción hacia el futuro cuando pronunció María está embarazada, también lo tiene suponer que la creencia previa del hablante en $(2 b)$ es hacia el futuro y que solo se refiere al ahora de la enunciación. Similarmente, así como la oración Juan dijo/ha dicho que María... dice que María está embarazada ahora y que también lo estaba antes, del mismo modo (2b) dice que el oyente está vivo ahora y que también lo estaba antes, cuando el hablante no tenía evidencia para afirmarlo. 
incluye el momento actual) has estado vivo, mientras que antes no tenía evidencia de que, durante todo ese mismo intervalo, lo estuvieras. Más argumentos a favor de la interpretación de (38) por sobre la de (37) para (2b) se verán en §5.2.1, cuando se presenten evidencias de que aún en (2b) la evidencia es indirecta.

Resulta de interés comparar la presente propuesta con una explicación de Ippolito (2004) sobre el equivalente italiano de lo que en español suele llamarse imperfecto citativo (¿Cómo te llamabas?): allí, según esta autora, el hablante no busca simplemente información, sino información vieja - de allí el tiempo pasado-, es decir, información que estaba disponible en otro tiempo y que ya no recuerda al momento de habla; un razonamiento similar, solo que reemplazando la búsqueda de información por la afirmación, es aplicable a las expresiones equivalentes a (2b) en español general, que también utilizan el imperfecto (;Estabas vivo!). Es cierto que aún queda por explicar por qué el pasado se expresa en un caso mediante imperfecto y en otro mediante algo semejante a un perfecto; de todos modos, esto no tiene nada de inusual, ya que es sabido que tanto perfectos como imperfectivos, pero no perfectivos, son compatibles translingüísticamente con la miratividad; véase DeLancey (1997), Aikhenvald (2004), y en especial Peterson (2010: 147 y passim) y Torres Bustamante (2013: 110ss). Nuestra conjetura al respecto es que lenguas como el español general, que utilizan el imperfecto, solo codifican una aseveración sobre el lapso previo al descubrimiento, mientras que la continuidad de la eventualidad hasta el presente es una implicatura. Es decir, la morfología de imperfecto es "real": solo se afirma algo sobre el pasado.

Retornando a la comparación con el perfecto, obsérvese que todo el esquema propuesto aquí para dar cuenta de ra'e/había sido presenta similitud con el del perfecto según la propuesta de Iatridou et al. (2003). En efecto, no solo pueden compararse los casos de ra'e/había sido en que la eventualidad culmina o cesa antes de TAE con los casos de perfecto existencial, como hemos hecho en (36), sino que también son comparables los casos en que la eventualidad se mantiene en curso hasta TAE con los casos de perfecto universal. En este segundo par es indispensable que la eventualidad no esté delimitada, a diferencia del primero, donde cesa o culmina antes del límite derecho del lapso, es decir, de TAE.

(39) ¡Reikove nipo ra’e! (= 2b) / ¡Había sido que estás vivo! (= 3b)

$$
\mathrm{TAE}(=\mathrm{TH})
$$

TEv: $\mathrm{xxx}$



lapso previo 
Nuevamente, TAE corresponde al límite derecho del lapso del perfecto, que a su vez corresponde aproximadamente a $\mathrm{R}$ de las versiones clásicas de la teoría de Reichenbach (o bien a TT, a condición de que allí TT se conceptualice como un punto y no un intervalo, concretamente el punto final de un intervalo). Lo crucial es que en (2b) TAE coincide con un subintervalo de TEv, de modo que queda explicado el efecto de evidencia directa, (véase \$5.2.1), a la vez que TAE coincide con TH, de modo que se da cuenta de la sorpresa y del efecto de recencia, véase §5.3.

Una diferencia esperable del gp ra'e respecto del perfecto de las lenguas europeas es que, mientras en estas últimas la ubicación del límite derecho del lapso del perfecto respecto de $\mathrm{TH}$ es determinada por el verbo finito - es decir, de coincidencia si se trata de pretérito perfecto, de precedencia si pluscuamperfecto y de posterioridad si futuro perfecto-, en gp hay una única forma y que vale para TAE presente y pasado, ya que esta lengua no gramaticaliza la distinción presente-pasado, y no se admite un TAE en futuro, ya que no puede codificarse una adquisición futura de evidencia. Lo mismo puede decirse del ep había sido, dado que es invariable y que la morfología de pluscuamperfecto es solo residual. En cuanto al límite izquierdo del lapso del perfecto, este puede ser especificado mediante adjuntos o contextualmente (Iatridou et al.2003: 166), y lo mismo sucede con ra'e/había sido respecto del límite izquierdo del intervalo previo a TAE: un ejemplo de límite especificado mediante adjuntos es pe márte 'el martes'en (34), mientras que un límite contextual podría ser el comienzo de cualquier lapso en el cual la expectativa previa del hablante sobre $p$ sea relevante, p. ej. el momento en que notó la ausencia del oyente en (2b).

Cerramos la sección con algunas precisiones sobre la relación entre TAE y los demás tiempos.

- El lapso previo no necesariamente incluye (parte de) TEv, ni necesariamente TEv es anterior a TAE o a TH: pruebas de esto pueden verse cuando ocurren el prospectivo u otras marcas de futuro relativo, donde TEv $>$ TAE/TH, cf. (35). Esto no representa un problema, ya que ra'e y había sido no dicen nada de la relación de TEv con otras referencias temporales. Lo que sucede en (35) es que -ta, como cualquier futuro relativo, sitúa el TEv con posterioridad a una referencia temporal TT; luego ra'e/había sido sitúa a TAE en TH. Para recapitular sobre la relación entre TEv y TAE: si la eventualidad es télica, TEv $<$ TAE, si es atélica, habitual o interviene el progresivo, TEv $\supset$ TAE, y si interviene el prospectivo, deóntico, inminencial u otros, TEv $>$ TAE.

- Si bien TAE se sitúa por defecto en TH, hay al menos tres tipos de casos en que esto no ocurre: en (7), el TEv de la principal sitúa en pasado el TAE, que ocurre en una subordinada; en (30), es el adjunto extraoracional asẽrõguare... 'cuando 
salí...' lo que sitúa TAE antes de TH, y en (31) lo hace el verbo de una oración yuxtapuesta a la que contiene ra'e/había sido, que actúa como contexto. Lo de (7) es un fenómeno bien conocido en las lenguas con tiempo como el español: en Encontré que un rayo habia partido el lapacho, el TT de la subordinada coincide con el TEv de la principal, que es pasado según lo indica el tiempo verbal; en gp, los hechos son similares si aplicamos a TAE lo dicho para el TT de la subordinada española, solo que allí no es el tiempo verbal quien sitúa al verbo principal en pasado, sino el contexto o adjuntos.

- No todo adjunto temporal toma alcance sobre ra'e/había sido "llevándose consigo" el TAE, como en (30). Ejemplo de esto es (34): pe márte 'el martes' establece un TT, pero TAE coincide con TH mientras que TT termina antes de TH. Nótese además que allí la relación entre TEv y TT indica una eventualidad no delimitada, lo cual prueba nuevamente que ra'e y había sido son compatibles con cualquier tipo de relación entre TEv y TT (véase §6.2).

\subsection{Evidencialidad}

\subsubsection{Siempre hay evidencia indirecta}

En la introducción a $§ 5$ afirmamos que la naturaleza de la evidencia no necesita ser explicitada en la definición de ra'e/había sido, aunque esta última implica que siempre hay evidencia indirecta. En las eventualidades no puntuales, esto es claro: dado que la naturaleza de la evidencia depende de la relación entre TEv y TAE -si se solapan es directa y si no indirecta- y que TAE es un punto, siempre habrá al menos un subintervalo de TEv que no se solape con TAE y, por lo tanto, evidencia indirecta. Esto se observa claramente en (2a): el hablante percibe en TAE una consecuencia de la eventualidad, p. ej. la presencia del oyente junto a él, y esto es a su vez evidencia indirecta de la eventualidad en sí: la venida previa del oyente. El mismo enunciado, en cambio, es infeliz si el hablante se halla en la puerta de su casa y ve llegar a su interlocutor por razones que veremos luego.

En cambio, (2b) parece a primera vista un caso de evidencia puramente directa. ¿Qué percibe el hablante allí? Que el oyente está vivo, lo que en sí mismo corresponde al contenido proposicional, de modo que la evidencia aparece como directa. Así, la correspondencia entre valores de presente y evidencia directa planteada en $\S 4.3$ recibiría una explicación sencilla: si la eventualidad se continúa hasta el presente, y si TAE coincide con el presente, entonces el oyente puede llegar a percibir la eventualidad de modo directo. Sin embargo, mientras que $p$ ra'e dice que el hablante 
recién en TAE percibe que $p$, el contenido de $p$, en cambio, es que el participante está vivo no solo en TAE, sino también en el lapso previo durante el cual el hablante no lo sabía, según se sostuvo en $§ 5.1$. De este modo, la evidencia puede entenderse como indirecta incluso en (2b): que el oyente esté vivo en TAE es evidencia indirecta de que ha estado vivo también durante un lapso mayor; una paráfrasis sería ;Has estado vivo (todo este tiempo que incluye el presente, sin que yo lo supiera hasta ahora)! En este sentido, ra'e contrasta con kuri, que es feliz en enunciados donde el hablante ha sido testigo directo de la eventualidad durante todo el intervalo, y no solo durante su límite derecho, cf. (23). Nuevamente puede trazarse una analogía con el perfecto universal: pese a que en este último el final de TEv coincide con TT/TH, no por eso se trata de un presente a secas, ya que el perfecto universal no dice solo que algo ocurre en TT/TH sino que venía ocurriendo antes ${ }^{20}$; análogamente, $p$ ra'e no dice solo que algo ocurre en TAE/TH sino que venía ocurriendo antes, de modo que (2b) no es comparable a un presente sino a un perfecto universal.

Existe un argumento más que sugiere que hay evidencia indirecta en $(2 b)$ y que la aserción involucra al intervalo previo y no solo a TAE: (2b) es compatible con nipo (nimbo, mbo), un modal/evidencial que, en ausencia de $r a^{\prime} e$, excluye la evidencia directa $^{21}$ :

(40) ??A-jú-ma nipo.

1sg.ACT-venir-ya PART

'??Por lo visto ya vine' (Comentario del informante: nipo es como que te contaron; ajúma nipo no puede ser, poque la acción la realizo yo. En caso de que sea él el que viene puede ser: oúma nipo 'él ya vino').

Si nipo es un evidencial indirecto, como sugiere (40), y aún así es gramatical en (2b), es porque allí no hay realmente evidencia directa de $p^{22}$.

Resta considerar aún lo que sucede cuando la eventualidad es puntual, ya que allí cabe en teoría la posibilidad de que TEv coincida por completo con TAE, de modo que no habría evidencia indirecta. Sin embargo, esto no se documenta: con ra'e/había sido,

20 Nótese que tomamos aquí a TT como equivalente a R y al límite derecho del lapso del perfecto, y no como equivalente a este último lapso.

21 Guasch \& Ortiz (1996) lo definen como "partícula o sufijo de potencial o duda", y para po, que consideran equivalente, proponen las glosas tal vez, quién sabe. Véase nota 28 sobre la segmentación de nipo.

22 Cierto es que en (2b) hay ironía y que podría atribuirse a ella el evidencial indirecto nipo, pero los mismos valores de aparente evidencia "directa" y "presente" se obtienen en una interpretación literal; véase también (47), donde el predicado es genérico. 
el TEv de una eventualidad puntual y télica como p. ej. oguahe 'llega' se interpreta como anterior a TAE, mientras que en una atélica como p. ej. overa 'relampaguea' es posible, además, una interpretación iterativa, de modo que TEv incluye a TAE. En los dos casos, entonces, queda al menos un subintervalo de TEv excluido de TAE.

¿A qué se debe la interpretación obligatoria de TEv < TAE en -guahe 'llegar'? Debe notarse que esto no es exclusivo de logros, sino de eventualidades télicas en general (cf. - ju 'venir' en (2a) ${ }^{23}$. Esto puede correlacionarse con el hecho de que, translingüísticamente, las culminaciones de eventualidades tienden a evitar la coincidencia con $\mathrm{TH}^{24}$, así como los perfectivos son incompatibles con las lecturas de presente. En consecuencia, si TH = TAE, entonces necesariamente se evita también la coincidencia de la culminación con TAE.

Esto no alcanza, sin embargo, para explicar la interpretación TEv $<$ TAE en los casos en que TAE y TH no coinciden, como (7), de modo que habría que conjeturar alguna tendencia más general a evitar la coincidencia de dos puntos temporales como lo son la culminación y TAE. Por otra parte, la razón puede buscarse también en la naturaleza misma del evento de adquisición de evidencia: esta última debe preexistir para que pueda ser percibida. Para p. ej. la lluvia, la evidencia puede consistir en la eventualidad misma (la lluvia, TEv $\supset$ TAE) o en un indicio de que ocurrió (el suelo mojado, TEv < TAE) u ocurrirá (las nubes negras, TEv > TAE), pero en ningún caso lo que provoca el cambio de estado mental puede comenzar a existir al mismo tiempo que el cambio mismo.

\subsubsection{Ra'e/había sido no son felices cuando hay evidencia directa de la culminación}

Debe explicarse todavía por qué (2a) es infeliz cuando hay evidencia directa de la llegada del oyente, ya que la definición propuesta no excluye, en principio, esa posibilidad. En efecto, asúmase que -ju 'venir' es, como su correlato español, una realización [accomplishment], esto es, una eventualidad durativa y télica. Entonces,

\footnotetext{
23 Aunque en elicitación se da a menudo 'llega' como traducción de oguahẽ (incluso en una oración completa), el valor de presente es mucho más raro en el habla espontánea, salvo cuando se acompaña del progresivo hina o tiene una lectura habitual.

24 Un referencista anónimo menciona los relatos de fútbol como posible contraejemplo, como p. ej. en $X$ se la pasa a $Y$, esto es, el llamado presente puntual o momentáneo. Con todo, creemos que esto no invalida la observación. Como se señala en RAE (2009: 1718), estos presentes "no describen hechos narrados a la vez que tienen lugar, sino recientes o inmediatos", con lo cual la culminación de la eventualidad nunca coincide con $\mathrm{TH}$, entendiendo $\mathrm{TH}$ como un punto.
} 
incluso si el hablante ve llegando a su interlocutor, puede existir aún un subintervalo de TEv del cual no tiene evidencia, como en (2b): el que incluye el viaje del participante y excluye su llegada. Pero (2a) no es feliz en tal contexto, y lo mismo sucede con cualquier eventualidad delimitada, cf. (41), lo que obliga a replantear las condiciones de felicidad de ra'e.

$\begin{array}{llll}\text { ¡Re-'u-pa } & \text { la } & \text { chipa } & \text { ra'e! } \\ \text { 2sg.ACT-comer-todo } & \text { DET } & \text { chipa } & R A^{\prime} E\end{array}$

‘i(Había sido que) te comiste toda la chipa!' (Feliz si p. ej. el hablante ve migas sobre la mesa, pero no si ve al oyente comerse el último pedazo).

La diferencia crucial entre -kove 'vivir', por un lado, y-ju 'venir' o - 'upa 'comerse (todo)', por otro, es que la primera eventualidad es atélica, y por ende homogénea, y las otras no. En cualquier subintervalo de la eventualidad de (2b), lo descripto por el verbo se verifica, mientras que esto no sucede en (2a) o (41): en cualquier subintervalo de ESTAR.VIVO(x) es cierto que $\mathrm{x}$ está vivo, mientras que en un subintervalo de VENIR(x) no es cierto que $\mathrm{x}$ haya venido, ni en un subintervalo de COMERSE( $\mathrm{x}, \mathrm{y})$ es cierto que $\mathrm{x}$ se haya comido y, ya que las eventualidades télicas -a menos que intervenga morfología imperfectiva- solo se verifican cuando han culminado. Esto es lo que hace infelices (2a) y (41) cuando la evidencia es directa. Precisamos entonces TAE y formulamos las condiciones de felicidad de $p$ ra'e/había sido en (42):

(42) a. TAE es la primera percepción que el hablante tiene de la eventualidad (incluyendo estados previos o posteriores), completa o no.

b. Pra'e/habia sido es feliz ssi $\mathrm{p}=1$ en TAE.

Cuando el hablante es testigo directo de la culminación de la eventualidad télica, las condiciones de (42) no se cumplen porque, en realidad, la primera percepción de la eventualidad -TAE- corresponde a un punto anterior a la culminación, en el cual aún $\mathrm{p}=0$; en (41), esto puede corresponder a un momento en el cual el participante estaba comiendo pero aún quedaba chipa. Nótese que (42) no implica que deba existir un subintervalo de TEv anterior a TAE, lo cual excluiría, de modo indeseable, casos de ra'e con futuro como (35). Al contrario, (42) explica satisfactoriamente (35): allí $p=1$ en TAE asumiendo una interpretación modal inercial para el prospectivo, de modo que es cierto que el hablante y su grupo se quedarán en los mundos en que todo ocurre según lo previsto, y TAE corresponde a la primera percepción de la eventualidad: el estado previo. Por otra parte, cabe mencionar una formulación alternativa a (42) que excluye la referencia a la primera percepción e introduce en cambio el intervalo previo: 
(43) Pra'e es feliz ssi $p=1$ en TAE y ssi existe un intervalo previo en el cual $p=1$ y el hablante no lo sabe.

Si el hablante ve al oyente llegando y exclama (2a) conceptualizando la eventualidad como incompleta, es decir, situando TAE antes de la llegada, la infelicidad se debe a que $p=0$ en TAE; si conceptualiza la eventualidad como terminada, es decir, considera que la llegada se produce mientras lo ve y por lo tanto sitúa TAE después, la infelicidad se debe a que no hay intervalo previo en el cual $p=1$ y él no lo supiera, ya que adquirió el conocimiento de inmediato. También (43) explica satisfactoriamente (35): existe un intervalo previo a TAE en el cual el hablante y su grupo están en el estado previo a quedarse sin que el hablante lo sepa ${ }^{25}$.

\subsubsection{Ra'e y había sido no son narrativos porque TAE siempre queda especificado}

La imposibilidad de utilizar ra'e/había sido como evidencial narrativo, mencionada en $\S 4.2$, se debe a que, a diferencia de otros evidenciales para los cuales también se ha postulado un TAE (Lee 2011, Smirnova 2011, Koev 2011), con ra'e/habia sido la localización temporal del TAE siempre resulta especificada, lo cual choca en usos narrativos, cf. (32) repetida abajo. En efecto, cuando TAE coincide con TH se identifica con este último y, en los casos en que TAE $<\mathrm{TH}$, como (7), (30) y (31), la principal, el adjunto temporal y el contexto -respectivamente- proveen la localización temporal precisa de TAE. En otras lenguas para las que se ha postulado TAE esto no siempre es así, cf. (32) con (44):

(32) Ñorairõ guasu o-ñepyrũ mil_noveciento_treinta_y_do-pe ra'e. guerra grande 3ACT-empezar mi_novecientos_treinta_y_dos-en $R A^{\prime}{ }^{\prime}$ '(Había sido que) la Guerra Grande empezó en 1932'. (Infeliz si se busca un uso narrativo del evidencial).

(44) Coreano (Lee 2011: 287)

Kucekkey pi-ka o-ass-te-la. anteayer lluvia-NOM caer-PAS-EV-DECL

'[Inferí que] había llovido anteayer', es decir, 'Llovió anteayer' (evidencia indirecta).

$25 \quad$ Una ventaja de (43) es que explica más económicamente por qué TEv no puede coincidir con TAE, es decir, por qué una eventualidades puntual como oguahẽ ra'e '(había sido que) llega' no es feliz con valor de presente y evidencia directa: porque no habría intervalo previo en el cual $p=1$. En cambio, con (42) debe estipularse que la fase previa es parte de la eventualidad, de modo que en el ejemplo anterior la primera percepción de la eventualidad no sería la llegada del participante sino la fase previa. De cualquier modo, como se expresó arriba en el texto, la imposibilidad de $\mathrm{TEv}=\mathrm{TAE}$ posiblemente pueda explicarse por principios más generales, sin necesidad de que se explicite en la definición de ra'e/había sido. 
Según Lee, el evidencial -te codifica que TAE precede a TH y, además, establece a TAE como el tiempo de evaluación de los operadores temporales. Además, -ess (aquí superficialmente -ass) codifica que TEv es anterior al tiempo de evaluación, que, por lo antedicho, es TAE en (44); en consecuencia, cuando -ess coocurre con -te, TEv $<\mathrm{TAE}$, por lo que la evidencia es indirecta. En (44) no puede precisarse el momento exacto en que el hablante adquiere la evidencia (p. ej. cuando ve el suelo mojado), el cual puede haber ocurrido en cualquier momento entre anteayer tras la lluvia y hoy, y los datos de Lee no sugieren que, para que (44) sea feliz, deba haber aparecido previamente en el discurso algún adjunto temporal que permita precisar TAE. Esto vuelve a -te, y a los evidenciales que admiten este análisis (cf. Smirnova [2011] para el búlgaro) compatibles con valores narrativos. En cambio, con ra'e y había sido, aún en los casos en que TAE $<\mathrm{TH}$, TAE es siempre precisable temporalmente, lo que los vuelve infelices en un uso narrativo. Entonces, proponemos que estas diferencias se deben a que en ep y gp TAE coincide por defecto con $\mathrm{TH}$, lo que hace que sea siempre identificable, mientras que en coreano lo precede y, en consecuencia, puede corresponder a cualquier punto no precisado dentro de un lapso anterior a TH y no anterior a TEv.

Más precisamente, y a fin de explicar también los casos en que TAE no coincide con TH, debe suponerse, siguiendo a Smirnova (2011), que en presencia de evidenciales como ra'e/había sido existen dos tiempos de evaluación: uno para la proposición bajo el alcance del evidencial, específicamente introducido por este último y que coincide con TAE o, más exactamente según Smirnova (2011: 288) con el ahora de quien sostiene la actitud hacia la proposición [attitude holder's now] y, por otro lado, un tiempo de evaluación de todo el enunciado $(t)$, que es TH por defecto pero que puede desplazarse al pasado. De acuerdo con esto, entonces, decimos que en gp y ep TAE siempre coincide con $t$-sea presente o pasado- mientras que en coreano lo precede.

Este análisis lleva a otra consideración: la diferente relación con $t$ que tienen ra'e/había sido y -te no es solo temporal sino, en cierto sentido, aspectual. En efecto, si comparamos a $t$ con el papel delimitador que juega TT en el aspecto y a TAE con el TEv de un predicado de percepción, resulta que ra'e/había sido tratan al evento de adquisición como una eventualidad perfectiva (i. e. incluida en el TT) mientras que con -te se asemeja a un perfecto, es decir, la eventualidad es anterior al TT: en oí que $\mathrm{p}$, en las variedades que distinguen entre aoristo y perfecto, el TEv normalmente puede precisarse en virtud de su inclusión en TT, mientras que este no es el caso en los usos experienciales de he oído que $\mathrm{p}^{26}$. Así como el perfecto no permite por sí

26 La analogía resulta oscurecida por el hecho de que TAE en ep y gp suele coincidir con el presente, que es incompatible con el perfectivo; atiéndase, entonces, solo a lo aspectual y no a lo temporal. 
mismo especificar temporalmente el TEv identificándolo con ningún otro tiempo -a menos que algún adjunto de TEv lo haga- mientras que el aoristo especifica TEv situándolo dentro de un TT fijado por el contexto o adjuntos, del mismo modo -te no permite especificar el TAE identificándolo con otro tiempo, mientras que ra'e/había sido siempre lo especifican identificándolo con $t$.

\subsection{Miratividad, "sorpresa diferida" y contraexpectativa}

El descubrimiento, contraexpectativa o falta de expectativa del hablante respecto de $p$ que se observan con ra'e y había sido quedan explicados por el hecho de que la evidencia está temporalizada: existe un tiempo en que el hablante no tenía evidencia de $p$ y quizá no creía que $p$ (o creía que $\neg p$ ), por lo cual $p$ llega como información no esperada en TAE.

Esto es independiente de dónde se localice TAE, es decir, coincidente con TH, como en el caso por defecto, o anterior a TH, como en los casos de (7), (30) y (31) tratados en $\$ 4.2$. En cambio, la sorpresa que se corresponde con la exclamación solo ocurre cuando TAE coincide con TH, de modo que el descubrimiento de $p$ es reciente y se satisface así restricción de recencia expuesta en $(11)^{27}$. De este modo, la restricción de recencia queda explicada aquí por la relación entre TAE y TH (cf. Smirnova 2011: nota 5).

Con todo, no parece que la superposición de TAE con TH garantice exclamación, ya que oraciones principales con ra'e/había sido pueden enunciarse sin entonación exclamativa ni partículas enfáticas, y no queda claro entonces que tales usos de ra'el había sido deban considerarse "mirativos" en el sentido de los evidenciales mirativos presentados en $\$ 2.3$, esto es, exclamativos, y no usos evidenciales "puros": por lo expuesto en el párrafo anterior, el mero descubrimiento de $p$ no puede confundirse con la sorpresa, ya que puede ocurrir descubrimiento sin sorpresa. Esto sugiere, entonces, que la sorpresa que expresan ra'e y había sido, cuando la expresan, tiene dos componentes independientes: por un lado, el descubrimiento de $p \mathrm{y}$, por otro, la evaluación de $p$ como sorpresiva, y que solo el primero está codificado en ra'e/había

27 Como se discutió en §5.2.1, la simultaneidad de dos puntos es problemática. Además, en los hechos el momento de adquisición suele preceder por instantes al de enunciación, por lo que los detalles de la expresión "TAE $=$ TH" requieren algún desarrollo: o bien TH es un intervalo que incluye los instantes previos (que aún deberían definirse) y por lo tanto a TAE, o bien TAE es el límite inicial de un intervalo que se extiende mientras dure el efecto novedoso de la adquisición (cf. [11]) o la disponibilidad de la evidencia, o bien TAE y TH son dos puntos contiguos, más que coincidentes, incluidos ambos en un intervalo común (¿TT?). 
sido. La restricción de recencia, entonces, expresaría una condición necesaria pero no suficiente para la expresión de sorpresa.

La interacción de las partículas niko, nipo y otras con ra'e (y aun había sido) merece mayor atención que la que podemos dedicar aquí. Sin embargo, parece ser que al menos niko se vincula con la fuerza ilocucionaria y no con la evidencialidad. El ejemplo (45a) muestra que ko/niko expresan contraexpectativa por sí mismas, pero aquí del oyente y no del hablante; su imposibilidad en subordinadas (cf. (45b-c)) es consistente con un análisis ilocucionario ${ }^{28}$.
a. ¡A-jú-ma ko/niko!
1sg.ACT-venir-ya PART
‘Ya vine'! (Comentario del hablante sobre ko: "porque no tenían fe en que yo pudiera venir”. Escuchado.)
b. ¡Ai-kuaa
1sg.ACT-saber
‘Sé que vino!'
ko/niko
o-u-ha-gue!
PART
3ACT-venir-COMP-PAS
c. *iAi-kuaa o-u-ha-gue ko/niko!
1sg.ACT-saber 3ACT-venir-COMP-PAS PART
Significado buscado: énfasis o contraexpectativa en la subordinada.

Esto presenta similitud con los datos del checheno de (14), donde la evidencialidad y la codificación de la sorpresa están manifestadas en exponentes diferentes: en gp, la evidencialidad/descubrimiento estaría expresada por ra'e y la sorpresa por ko/niko.

Por último, ra'e y había sido no necesariamente involucran contraexpectativa, como ilustra (46):

(46) ¡Re-jahu ngo hína ra'e!

‘(Había sido que) te estabas bañando!' (Contexto: el hablante había estado llamando por teléfono a su interlocutor y este no atendía).

Aquí no se trata de que el hablante esperara que el oyente no se estuviera bañando. Más bien, no tenía ninguna expectativa respecto del baño, y la adquisición de evidencia sobre $p$ aparece súbitamente como una explicación de que no le contestaran el teléfono.

28 Proponemos analizar niko, nipo como $n a+k o, n a+(i) p o$, respectivamente. $N a$ es un enclítico de cortesía o empatía que puede acompañar imperativos ('por favor') y exclamaciones (angá-na ‘ipobrecito-na!'). Considerando la caracterización de po hecha en torno a (40), su análisis como modal sí parece plausible; de todos modos, nipo incluye también la partícula na, muy probablemente ilocucionaria. 
Es cierto que aún resulta válida la explicación propuesta por Torres Bustamante (2012, 2013) para el pluscuamperfecto andino: los mundos en que el oyente no se estaba bañando son mejores que aquellos en los que sí, ya que la posibilidad del baño no estaba presente en la mente del hablante. De todos modos, no parece adecuado llamar a esto contraexpectativa sino más bien falta de expectativa [unexpectedness]: en la contraexpectativa, el hablante espera que $\neg p$, mientras que aquí el hablante no tiene nada en mente respecto de $p$, esto es, ni $p$ ni $\neg p$. En la contraexpectativa, se elige la menos probable de dos opciones contradictorias ( $p$ frente a $\neg p$ ), mientras que en (46) se elige una entre varias opciones: $p$ frente a $q, r, s$, etc.: la razón de que no me atendiera es que se estaba bañando, no que no deseara atenderme, que estuviera ausente, que se sintiera mal, etc. Si la propuesta de Torres Bustamante sugiere un paralelismo entre los evidenciales mirativos y el foco contrastivo, (46) muestra que al menos ra'e y habia sido pueden relacionarse también con el foco neutro. De aquí puede desprenderse también otra consideración: si la sorpresa (del hablante o del oyente) implica un mecanismo similar al del foco (selección de $p$ frente a $\neg p$, o frente a $q, r, s$ ), entonces el $k o$ indicador de sorpresa de (45)-(46) no sería otra cosa que un uso especial de un marcador de foco.

\subsection{Sumario}

A lo largo de $\S 5$ se ha dado respuesta a las preguntas (a-c) planteadas al final de $\S 4$. En cuanto a (a), los casos de aparente evidencia directa y referencia presente ocurren cuando la eventualidad es no delimitada y por ende llega a superponerse con TAE. La imposibilidad de usar ra'e/había sido como evidencial narrativo -pregunta (b)obedece a que TAE siempre es especificable, y respecto de (c), la sorpresa equiparable a la exclamación solo tiene lugar cuando TAE se superpone a TH. En cambio, la pregunta (d), por qué ra'e no parece codificar adquisición de evidencia en preguntas y bajo modales, ha quedado sin responder. Esto, junto con otras cuestiones pendientes, se discute en la sección siguiente.

\section{DISCUSIÓN Y CUESTIONES PENDIENTES}

\subsection{Ra'e en preguntas y contextos modales}

En $\S 1$ y $\S 4$ se presentaron casos en los que no es claro que ra'e denote adquisición de la evidencia: preguntas (4), declarativas bajo el conjetural/inferencial -ne (5) y apódosis de condicionales (6). En todos ellos ra'e actúa debajo de algún operador modal o 
ilocucionario y no se corresponde con había sido en ep. Esta aparente ausencia de valor evidencial podría sugerir que ra'e fuera allí un simple operador temporal que ligaría una variable similar a $\mathrm{R}$ en la teoría clásica de Reichenbach, más bien que un TAE; en términos de Torres Bustamante (véase §2.5), el argumento temporal con valor [pasado] sería interpretado propiamente en ST/SFlex y no en SComp. Sin embargo, otros indicios sugieren que allí el valor de ra'e no difiere esencialmente del que tiene en declarativas, según veremos.
a. ¿Moõ-pa
re-1̃
ra'e?
dónde-PART
2sg.ACT-estar
$R A ' E$
¿Dónde has estado/estuviste/estabas?'

b. ¿Moõ-pa re-ĩ?

dónde-PART 2sg.ACT-estar

‘¿Dónde estás?'

(5)

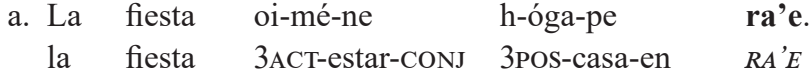
'La fiesta habrá sido en su casa'. (Conjetura o inferencia)
$\begin{array}{cclll}\text { b. La fiesta } & \text { oi-mé-ne } & \text { h-óga-pe } & \text { hína. } \\ \text { la } & \text { fiesta } & \text { 3ACT-estar-CONJ } & \text { 3POS-casa-en } & \text { PROG }\end{array}$
'La fiesta será en su casa'. (Conjetura o inferencia)

(6)
a. Oi-kuaa rire,
he'i-mo'ã
3ACT-saber
si.PAS
3ACT.decir-MOD
ndéve ra'e.
'Si lo \{supiera/hubiera sabido\} te lo habría dicho'.
b. Oi-kuaa rire,
3ACT-saber si.PAS
he'i-mo'ã
ndéve.
3ACT.decir-MOD 2sg.OBL
'Si lo supiera te lo diría'.

Una característica común a los casos de (4)-(6) es que en ellos las lecturas de pasado son preferidas incluso con predicados no delimitados, es decir, se eluden las lecturas universales como la esquematizada en (39). Sobre esto último, una explicación informal plausible es la siguiente: si la lectura universal implica que TEv llega a superponerse a TAE y que por lo tanto hay evidencia directa del final de TEv, entonces hay un choque con el modal/interrogativo: resulta extraño preguntar por o conjeturar sobre algo de lo que se tiene evidencia directa, y simplemente contradictorio combinar la apódosis de un condicional contrafáctico con la evidencia directa. Entonces, las preguntas, conjeturas y apódosis deben excluir el final de TEv, resultando así la lectura existencial y de pasado. De este modo, según este análisis, $r a$ 'e sí introduciría una variable temporal vinculada con la evidencia, pese a todo. 
Lo anterior predice también, acertadamente, que en los casos en cuestión debe esperarse ra'e como medio de expresión del pasado relativo y no kuri, que también puede cumplir tal función (véase §3 in fine, y también Tonhauser 2009, 2011), solo que cuando el hablante ha atestiguado la eventualidad completa. Según vimos arriba, en (4)-(6) solo resulta natural la evidencia indirecta, que en declarativas tratamos como epifenómeno de la no coincidencia entre TEv y el punto TAE. Kuri, en cambio, puede verse como un morfema que introduce un intervalo que incluye TEv, de modo que la evidencia directa se explicaría, también allí, por la coincidencia de TEv con una variable temporal, solo que ahora sería un intervalo en vez de un punto. Entonces, así como la interpretación universal de $r a$ 'e resulta contradictoria en (4)-(6) porque produce una evidencia indirecta indeseada, también debería serlo la presencia de kuri en lugar de ra'e, ya que kuri denotaría que se ha atestiguado toda la eventualidad, y no solo su final. Y efectivamente, kuri es infeliz en un condicional contrafáctico ${ }^{29} \mathrm{y}$, como mínimo, extraño en preguntas y, sobre todo, con -ne (los juicios varían algo de un hablante a otro en nuestros datos, pero en todos los casos se detecta anomalía y se prefiere ra'e, además de que allí kuri es virtualmente inexistente en producción espontánea). De este modo, dado que solo ra'e resulta "natural" en estos contextos, la oposición con kuri queda neutralizada, produciéndose así la ilusión de que ra'e solo manifiesta un valor tempoaspectual. Sin embargo, como hemos visto, aún puede sostenerse que allí ra'e introduce un punto temporal cuya coincidencia con TEv da lugar a un efecto de evidencia directa y su no coincidencia lo contrario, del mismo modo que en declarativas.

En conclusión, aun cuando los detalles de la semántica de ra'e en los contextos abordados en esta sección requieran mucho mayor estudio (especialmente en preguntas), también en ellos puede afirmarse que ra'e codifica evidencialidad.

\subsection{Ra'e/había sido en oraciones genéricas}

Ra'e y habia sido son compatibles con oraciones genéricas como la de (47) (véase también (9a)), lo que representa un problema para la analogía con el perfecto dada la asociación que suele establecerse entre imperfectivo y oraciones genéricas, por un lado, y entre perfect(iv)o y oraciones episódicas por otro (véase discusión en Torres

${ }_{29} \quad$ Es interesante notar que un condicional de pasado con rire y con el modal mo 'ã en la apódosis, que normalmente es contrafáctico, con kuri resulta fáctico, cf. (i), donde el condicional es epistémico.
(i) Ha-'e
rire
kóva, che-japu
mo'ã kuri.
1sg.ACT-decir si.PAS
DET 1sg.INCT-mentir
MOD KURI
'Si te dije eso, mentí'. 
Bustamante 2013: 68-69). Si TAE delimita la eventualidad estableciéndose como su límite derecho, del mismo modo en que lo hace R/TT en el perfecto, la continuación de TEv luego de TAE en (2b) es solo una inferencia pragmática, pero en oraciones como (47) la eventualidad no está delimitada.

(47) Lo rico r-a'y nimbo o-ti avei ra'e. DET.pl rico REL-hijo PART 3.ACT-tener_vergüenza también $R A$ 'E '(Había sido que) los hijos de los ricos también tienen vergüenza'. (Melià et al. 1958: 70)

Lo que ocurre en realidad es que TAE no delimita necesariamente el predicado, y en este sentido se rompe la analogía con el perfecto. Tampoco lo hace TT en los casos en que TT $\nsupseteq$ TAE, como muestra (34), donde es posible una interpretación imperfectiva. En ep, donde la oposición perfectivo-imperfectivo es mucho más consistente, resulta aún más claro que había sido es compatible con cualquier tipo aspectual, incluso imperfectivo (había sido que estabas acá). Todo esto sugiere que, como se propuso en §5.1, ra'e y había sido operan por encima de la relación entre TEv y TT.

\subsection{Ra'e/había sido vs. el pluscuamperfecto de contacto con quechua: referencia "presente" con predicados de nivel de estadio}

Como se expuso en $\S 2.5$, el español de contacto con quechua del sur de los Andes y el NO argentino utiliza el pluscuamperfecto con valores similares a los de ra'e/había sido. En esta variedad también los predicados estativos dan lugar a casos de aparente evidencia directa y referencia temporal presente. La explicación que propone Torres Bustamante $(2012,2013)$ es que esto obedece a propiedades de los predicados de nivel de individuo, ya que en esta variedad solo ellos dan lugar a lecturas de presente y evidencia directa, cf. (48a) con (48b) (extraídos de Torres Bustamante 2012: 360-361; 2013: 76-78).

(48) a. ¡Juan había sido alto!

‘(Resultó ser que) Juan es alto'. (Interpretación por defecto al ver a Juan parado)

b. ¡Juan había estado feliz!

'(Resultó ser que) Juan estuvo/estaba feliz!' (Extraña si Juan está sonriendo en el momento de habla)

Esta autora asume, al igual que nosotros para ra'e/había sido, que los pluscuamperfectos mirativos andinos realizan aserciones sobre el pasado; la lectura presente de (48a) es explicada por ella como una implicatura surgida por la interacción con la 
evidencia que el contexto provee (el hablante está viendo a Juan) y los "efectos de tiempo de vida" [lifetime effects] producidos por los predicados de nivel de individuo como ser alto. En efecto, dado que ser alto se sostiene durante toda la vida del individuo, la implicatura usual con morfología de pasado es que Juan ya no vive; sin embargo, dado que el contexto de (48a) no permite esto y tampoco se provee otro tiempo tópico contextual (como p. ej. en Me presentaron a Juan. ;Era alto nomás!), se fuerza entonces la interpretación en presente. Como esto no sucede con predicados de nivel de estadio como el de (48b), allí solo se obtiene la lectura de pasado.

Sin embargo, ra'e y había sido son compatibles con evidencia directa y lectura de presente incluso con predicados de nivel de estadio, como muestran (2b) y (49):

(49) ¡Juan o-vy'a ngo ra'e!

Juan 3ACT-estar_feliz PART RA'E

¡(Había sido que) Juan está feliz! (P. ej. si el hablante esperaba que Juan viniera triste y al llegar lo ve sonriendo).

La explicación de Torres Bustamante para el español andino no funciona entonces para el gp/ep. Sin embargo, a la inversa, la explicación que proponemos aquí para el gp/ep sí podría funcionar para el español andino con alguna estipulación. En efecto, nuestra explicación es que las "excepciones" como la (2b) se deben a una interpretación análoga a la del perfecto universal; pues bien, esta lectura es obligatoria para un predicado de nivel de individuo en contextos como el de (48a): necesariamente, en todo subintervalo del intervalo previo a TAE Juan fue alto. En cambio, por algún motivo, el pluscuamperfecto andino no admite lecturas universales con predicados de nivel de estadio.

\subsection{Ra'e/había sido vs. los perfectos evidenciales balcánicos}

Ra'e y había sido presentan diferencias importantes respecto de los perfectos evidenciales documentados en los balcanes. En primer lugar, en estos es necesario que TT coincida con $\mathrm{TH}$, es decir, solo funcionan como evidenciales en configuraciones equivalentes a un perfecto compuesto español; cuando TT $<\mathrm{TH}$, en cambio, la interpretación evidencial no se obtiene (Izvorski 1997), cf. (50a-b). En gp y ep, en cambio, ra'e y había sido ocurren también cuando $\mathrm{TT}<\mathrm{TH}$, según hemos visto en los casos de sorpresa "diferida", (7) y (30). 
(50) Turco (Izvorski 1997: 221-2)

a. Gel-miş-im.

venir-PERF-1sg

'Por lo visto vine'.

b. Gel-miş-tim.

venir-PERF-1sg.PAS

‘Había venido’ (infeliz como ‘por lo visto vine/había venido').

En segundo lugar, en el evidencial balcánico necesariamente TEv es anterior a TT, que a su vez coincide con TH; en la explicación de Izvorski (1997), que TEv esté excluido de TT y por ende de TH es lo que permite que la lectura evidencial tenga lugar. En gp y ep, en cambio, hemos visto que TEv puede solaparse con TT y TH, algo que ocurre regularmente cuando el predicado es no delimitado, véase nuevamente (2b) y (49).

En tercer lugar, el perfecto evidencial balcánico o, al menos, el búlgaro, no permite realizar inferencias hacia el futuro de acuerdo con Smirnova (2011), cf. (51), donde la forma verbal es equivalente a un futuro perfecto español.

(51) [Contexto de inferencia: el hablante vio el cielo nublado a la mañana y produce el enunciado a la tarde, cuando aún cree en su inferencia]

\# Štjalo da vali dovečera.

FUT.PERF COMP llover.IMPF.3sg.PRES esta_noche

Significado buscado: 'Va a llover esta noche' (inferencia). Feliz en una lectura reportativa. (Smirnova 2011: 288)

En (51) el TAE se sitúa en el pasado, según indica el contexto, mientras que el futuro perfecto localiza la lluvia en el futuro. Smirnova señala que (51) viola la máxima de cantidad: el hablante cree aún en TH que va a llover, pero sitúa la creencia en el pasado. En síntesis, en este análisis la imposibilidad de hacer inferencias hacia el futuro obedece a que, en (51), TAE $<\mathrm{TH}^{30}$. En gp y ep, en cambio, como TAE $=\mathrm{TH}-$ al menos en el caso por defecto-, las inferencias al futuro sí son posibles, como muestra (35). (Nótese de paso que el gp y el ep difieren del búlgaro en la morfosintaxis: la marca de futuro se liga al verbo léxico, no al evidencial ra'e/había sido).

30 Que (51) sea feliz con lectura reportativa se explica, según Smirnova (2011: 290), por lo siguiente: si lo que importa es el reporte de un tercero, que se remita a ese reporte en el momento de habla no viola la máxima de cantidad, ya que el hablante ignora cuál es la creencia de ese tercero en el momento de habla. Por otra parte, Smirnova excluye del análisis, en el artículo citado, los casos en que TAE $=\mathrm{TH}$ y que típicamente adoptan valor mirativo (sorpresa). Es de suponer que en ellos 


\subsection{Ra'e vs. raka'e}

Hemos explicado tanto la posibilidad de usos mirativos de ra'e y había sido como su imposibilidad de usos narrativos por la coincidencia de TAE con el tiempo de evaluación (por defecto, TH). Pues bien, sucede que raka'e, similar a ra'e en otros aspectos (véase $\$ 3$ in fine), es incompatible con usos mirativos a la vez que puede aparecer en narraciones, por lo cual suponer que codifica por defecto TAE $<$ TH es más que razonable. En (52) se muestra un ejemplo del mbyá, donde raka'e aparece con frecuencia en relatos míticos e históricos; allí, según este análisis, el hablante ha adquirido evidencia indirecta de lo narrado claramente después de que esto ha ocurrido pero antes del momento de habla, es decir, un análisis similar al que hemos visto en (44) para el coreano -te. Muchos otros aspectos de raka'e requieren mayor estudio, como su valor en preguntas y el valor de pasado remoto que le atribuyen las descripciones, pero al menos el análisis general que supone TAE $<\mathrm{TH}$ parece estar en el camino correcto.
(52)
$\begin{array}{lllll}\text { Mimby-'i ma tuja-kue-'i } & \text { ha'e } & \text { guaimi-gue-'i } & \text { o-mo-ñe'ẽ } \\ \text { flauta-DIM ya viejo-PL -DIM y } & \text { vieja-PL-DIM } & \text { 3ACT-CAUS-h }\end{array}$
'Antiguamente, los viejitos y viejitas tocaban la flauta'.
(Dooley 2006: 52)

\section{RA'E Y HABÍA SIDO. PERFECTO Y PLUSCUAMPERFECTO EVIDENCIAL EN ESPAÑOL SUDAMERICANO}

$R a^{\prime} e$ se corresponde en la mayoría de los contextos con había sido (que). ¿Por qué el ep habría "elegido" una forma de la perífrasis haber + participio para expresar valores evidenciales y mirativos? Las consideraciones presentadas en las secciones precedentes, especialmente en $\S 5.1$, dan una idea de la conexión entre tales valores de ra'e y el tiempo relativo expresado por la perífrasis con haber; además, las conexiones entre evidenciales/mirativos y formas de perfecto están abundantemente documentadas en otras lenguas, según hemos visto.

Ahora bien, ¿por qué para ra'e una forma fosilizada del pluscuamperfecto y no del perfecto compuesto, que sería lo esperable de acuerdo con el análisis propuesto si se establece una analogía entre TAE y TT (o R)? En efecto, si sostenemos que ra'e expresa $\mathrm{TAE}=\mathrm{TH}$ por defecto, $\mathrm{y}$ dado que el esquema del perfecto es TEv $<\mathrm{TT}=$ $\mathrm{TH}$, entonces al sustituir TT por TAE obtenemos un esquema similar al del perfecto

las inferencias a futuro sí son posibles, a condición de que p. ej. el hablante esté viendo el cielo nublado al momento de habla. 
en los casos -mayoritarios- en que TEv $<$ TAE. El uso del perfecto compuesto para expresar valores similares a los de $r a^{\prime} e$ se documenta efectivamente en el español de los Andes ecuatorianos, variedad de contacto con el quechua. Haboud (2008) señala que ese tiempo expresa que el hablante no se compromete con la veracidad de lo dicho: Anoche me encontré con la Pao... no me vas a creer esto, me contó que la Vero se ha casado (2008:176; cf. (7)). Olbertz (2009: 75) muestra asimismo que esta forma puede codificar valor mirativo: ¡No ha sido difícil! ‘ No es difícil!' [luego de escuchar instrucciones para llenar un formulario]; allí, el perfecto puede tener (aparente) referencia presente, como ep había sido ${ }^{31}$.

El pluscuamperfecto sería esperable, en cambio, si se supusiera $\mathrm{TAE}<\mathrm{TH}$, ya que entonces se obtendría un esquema similar al del pluscuamperfecto del español general equiparando TAE con TT (o R). De hecho, TAE $<$ TH es el valor por defecto que Smirnova (2011) asigna al perfecto evidencial del búlgaro, y más que razonablemente esto puede considerarse para el español de zonas andinas de Perú y Bolivia y del NO argentino, variedades de contacto con el quechua y/o el aymara. En ellas, el pluscuamperfecto no solo funciona como un evidencial mirativo (19), sino que también expresa evidencia indirecta adquirida antes del TH, es decir, toma valor narrativo:

(53) a. Español de Santiago del Estero (Vidal de Battini 1980: 391, III)

Y entonces advierte él que andaba arriba un cuervo y dice: “¿Sabe que me voy a hacer el muerto?”. Y así había hecho ['hizo’]. Se había tirado ['tiró'] en el campito y había dado ['dio'] vuelta al sieso.

b. Español de La Paz (Martín 1976: 119)

A: Sapir fue un gran lingüista.

B: ¿Cómo lo sabes?

A: Los libros lo dicen.

B: Entonces no puedes hablar así; debes decir: Sapir había sido un gran lingüista.

En estas variedades, todo sugiere que, en la transferencia desde la lengua indígena, el esquema probablemente más frecuente $\mathrm{TAE}<\mathrm{TH}$ se tomó como base para la adopción de la morfología española, y esta se generalizó a los casos de TAE $=\mathrm{TH}$, típicamente mirativos (en el sentido de sorpresa). En la variedad ecuatoriana de contacto, por su parte, el punto de partida habría sido $\mathrm{TAE}=\mathrm{TH}$.

En gp, en cambio, no existe una única forma para los casos en que TAE coincide con el tiempo de evaluación y aquellos en que lo precede, sino dos, ra'e y raka'e

31 El pluscuamperfecto en esta variedad, en cambio, expresa un evidencial indirecto de pasado según Kany (1970: 208), de modo que no admite la referencia presente, a diferencia del español andino de Perú, Bolivia y NO de Argentina. 
respectivamente. $\mathrm{Y}$, de acuerdo a lo esperado, esta última también puede transferirse al ep con el pluscuamperfecto, cf. el siguiente fragmento de la entrada $R A K A$ ' $E$ en el diccionario de Guasch \& Ortiz (1996): "reime raka'e: estabas, ya estabas (en buen castellano, no 'habías estado', como dice el paraguayo)"; cf. también RACA-E en Ortiz Mayans (1973): “oyapóraca-é [=ojapo raka'e]: había hecho”.

En cuanto a ra'e, sin embargo, dado que el valor más común es por lejos TAE $=\mathrm{TH}$, el pluscuamperfecto resulta inesperado, y una generalización del pluscuamperfecto desde raka'e a ra'e no es fácil de justificar. En suma, la "elección" del pluscuamperfecto como equivalente de ra'e permanece como algo que no podemos explicar. La cuestión queda pendiente de un mejor conocimiento sobre el ep colonial, incluyendo el peso del contacto temprano entre el Paraguay y el área andina ${ }^{32}$. Más aun, la amplia distribución de la estructura había sido + adjetivo con valor mirativo, que va desde Colombia al Uruguay (véase nota 2; Kany 1970; Avellana 2013) sugiere que no deben descartarse posibles tendencias del español peninsular que luego se vieran extendidas en América tras el contacto con lenguas indígenas.

\section{CONCLUSIONES}

A lo largo de este artículo hemos mostrado que la aparente polisemia de ra'e puede explicarse satisfactoriamente suponiendo que codifica la existencia de un tiempo de adquisición de la evidencia que, por defecto, coincide con el tiempo de habla, de modo que sus valores temporales, evidenciales y mirativos se explican por interacción de esto con otros factores. Además, ra'e implica o quizá entraña un intervalo previo durante el cual el hablante no tenía evidencia de $p$. Esto vale también para había sido, de distribución más reducida.

$R a$ 'e y había sido no especifican si la evidencia es directa o indirecta. La naturaleza de la evidencia depende, en cambio, de la relación entre TAE y TEv: si se solapan es directa y, si no, indirecta. Hemos mostrado además que, aun en los casos en que existe evidencia directa de un subintervalo de TEv, siempre existe también evidencia indirecta, ya que para que ra'e y había sido sean felices es que haya al menos un subintervalo de TEv que no coincida con TAE. Esto resuelve aparentes excepciones que se presentan si se analiza ra'e y había sido como evidencial indirecto liso y llano.

32 El Paraguay dependió administrativamente de Lima desde 1542 hasta 1776, año en que pasó a integrar el Virreinato del Río de la Plata. Sin embargo, hay amplio consenso sobre el aislamiento cultural y demográfico del Paraguay colonial ya desde la fundación de Asunción en 1537, por lo que la explicación el ep había sido a partir de contacto con el español andino requeriría bastante justificación. 
Si ha de seguir llamándose a esto "evidencial", debe entonces ampliarse la definición de esto último para incluir elementos que no codifiquen la fuente, ni siquiera su naturaleza, como ya ha sido sugerido p. ej. en Koev (2011).

Además, el requerimiento de que algún subintervalo de TEv quede "fuera" de TAE da una pista acerca de por qué los morfemas del tipo de ra'e y había sido son interlingüísticamente compatibles con morfología de perfecto e imperfectivo pero no de perfectivo: cuando perfectos e imperfectivos expresan evidencialidad, TAE asume el lugar de TT, de modo que siempre queda un subintervalo de TEv fuera de TAE; los perfectivos no pueden hacer esto, ya que en ellos TEv $-\mathrm{O}$ al menos su culminación- está incluido en TT.

El valor de pasado tampoco está entrañado en ra'e y mucho menos en había sido, que no presenta casos de aparente "pasado puro". Estos últimos se explican, para $r a$ 'e, por restricciones sobre la superposición de TEv con TAE y, por ende con TH, en ciertos contextos como preguntas, apódosis y oraciones conjeturales, algo que sí es posible en ausencia de ra'e con predicados no delimitados.

En cuanto a la miratividad, hemos visto que esta es comprendida por unos autores como 'descubrimiento' o nociones semejantes y, por otros, como sorpresa. El "descubrimiento" se explica, en la presente propuesta, por la noción misma de 'adquisición de la evidencia'. La sorpresa, por su parte, es un efecto de la interacción del "descubrimiento", cuando este coincide con TH, con la exclamación y, en gp, posiblemente también ciertas partículas como ko y niko.

Ra'e y habia sido son similares a muchos otros marcadores descriptos en otras lenguas en los que se cruzan la evidencialidad, el tiempo y la miratividad, pero tienen una particularidad: TAE siempre coincide con el tiempo de evaluación, mientras que en sus equivalentes en otras lenguas puede también precederlo (para esto último, el guaraní tiene un exponente especial: raka'e). Esto hace que ra'e y había sido no puedan funcionar como evidenciales narrativos: al coincidir TAE con el tiempo de evaluación, necesariamente expresan descubrimiento repentino, algo incompatible con los usos narrativos.

Por último, si bien la correspondencia de ra'e con una forma de la perífrasis haber + participio es consistente con el análisis propuesto, no podemos explicar que esa forma sea el pluscuamperfecto y no el perfecto. 


\section{BIBLIOGRAFÍA}

Abusch, D. (1997): "Sequence of Tense and Temporal de re", Linguistics and Philosophy 20 (1), pp. 1-50. http://dx.doi.org/10.1023/A:1005331423820

AdelaAR, W. (1977): Tarma Quechua. Leiden: Brill.

Aikhenvald, A. (2004): Evidentiality. Oxford: Oxford University Press.

Avellana, A. (2013). "Fenómenos de transferencia entre lenguas: evidencialidad en el español en contacto con el guaraní y el quechua", Estudios de Lingüística de la Universidad de Alicante (ELUA) 27, pp. 31-60. https://doi.org/10.14198/ elua2013.27.02

Carol, J. \& A. Salanova (2018): "Frustratives and viewpoint". Ponencia presentada en SULA 10, 4-6 de mayo.

Cerrón Palomino, R. (1994): Quechumara. La Paz: Centro de Investigación y Promoción del Campesinado.

Cerno, L. (2010): Descripción fonológica y morfosintáctica de una variedad de la lengua guaraní hablada en la provincia de Corrientes (Argentina). Rosario: Universidad Nacional de Rosario, tesis doctoral.

Cerno, L. (2010): "Evidencias de diferenciación dialectal del guaraní correntino", Cadernos de Etnolingüistica 3/2, pp. 1-7.

Cinque, G. (1999). Adverbs and functional heads: A cross-linguistic perspective. Oxford, Oxford University Press.

Cusinuamán, A. (1976). Gramática quechua: Cuzco-Collao. Lima: Instituto de Estudios Peruanos.

Davis, C., C. Potts \& H. Rullman (2007): "The Pragmatic Values of Evidential Sentences", in Proceedings of SALT 17. Ithaca: Cornell University, pp. 71-88. http://dx.doi.org/10.3765/salt.v17i0.2966

DE GRANDA, G. (1994): "Dos procesos de transferencia gramatical de lenguas amerindias (quechua/aru y guaraní) al español andino y al español paraguayo. Los elementos validadores", Revista de Filología Hispánica 74/1-2, pp. 127-141.

DE HAAN, F. (1999): "Evidentiality and epistemic modality: Setting boundaries", Southwest Journal of Linguistics 18/1, pp. 83-101.

DeLancey, S. (1997): "Mirativity: The grammatical marking of unexpected information”, Linguistic Typology 1, pp. 33-52. http://dx.doi.org/10.1515/lity.1997.1.1.33

DeLancey, S. (2001): “The mirative and evidentiality", Journal of Pragmatics 33 (3), pp. 369-382. https://doi.org/10.1016/S0378-2166(01)80001-1 
Dickinson, C. (2000): "Mirativity in Tsafiki”, Studies in Language 24, pp. 379-422. http://dx.doi.org/10.1075/s1.24.2.06dic

DiETRich, W. (2002): "La situación actual del contacto lingüístico guaraní-castellano en la Argentina", in N. Díaz, R. Ludwig \& S. Pfänder (eds.): La Romania americana. Procesos lingüisticos en situaciones de contacto. Madrid/Frankfurt: Iberoamericana/Vervuert, pp. 41-50.

DieTRICH, W. (2010): “Tiempo, aspecto y evidencialidad en guaraní", LIAMES 10, pp. 67-83. https://doi.org/10.20396/liames.v10i1.1511

DolZANI, V. (2016): "La partícula ra'e en guaraní mbyá. Temporalidad y evidencialidad", LIAMES 16 (2), pp. 241-260. http://dx.doi.org/10.20396/liames. v16i2.8646226

Dooley, R. A. (2006): Léxico Guarani dialeto Mbya com informações úteis para o ensino médio a aprendizagem e a pesquisa linguistica. http://www.sil.org.

Estigarribia, B. (2015): “Guaraní-Spanish Jopara Mixing in a Paraguayan Novel”, Journal of Language Contact 8, pp. 183-222.

https://doi.org/10.1163/19552629-00802002

FALler, M. (2002): Semantics and Pragmatics of Evidentials in Cuzco Quechua. Palo Alto: University of Stanford, tesis doctoral.

FALler, M. (2004): “The Deictic Core of 'Non-Experienced Past' in Cuzco Quechua”, Journal of Semantics 21, pp. 45-85. http://dx.doi.org/10.1093/jos/21.1.45

FLECK, D. W. (2007): "Evidentiality and double tense in Matses", Language 83/3, pp. 589-614. http://dx.doi.org/10.1353/lan.2007.0113

Giongi, A. \& F. PiAnesi (1997): Tense and Aspect: From Semantics to Morphosyntax. Oxford: Oxford University Press.

Gregores, E. \& J. A. SuÁrez (1967): A Description of Colloquial Guaraní. La Haya: Mouton de Gruyter.

Guasch, A. (1956): El idioma guaraní. Gramática y antología de prosa y verso. Asunción: CEPAG.

Guasch, A. \& D. Ortiz (1996): Diccionario Castellano-Guarani y Guarani-Castellano. Asunción: CEPAG.

Gynan, S. N. (2001): "Language planning and policy in Paraguay", Current Issues in Language Planning 2/1, pp. 53-118. https://doi.org/10.1080/14664200108668019

Haboud, M. (2008): “Ecuador”, in A. Palacios Alcaine (coord.): El español en América. Contactos lingüisticos en Hispanoamérica. Barcelona: Ariel, pp. 161-188. 
IATRIDOU, S. (2000): "The Grammatical Ingredients of Counterfactuality", Linguistic Inquiry 31/2, pp. 231-270. http://dx.doi.org/10.1162/002438900554352

Iatridou, S., E. Anagnostopoulou \& R. Pancheva (2003): “Observations about the Form and Meaning of the Perfect", in A. Alexiadou, M. Rathert \& A. von Stechow (eds.): Perfect Explorations. Berlín/Nueva York: Mouton de Gruyter, pp. 153-204. http://dx.doi.org/10.1515/9783110902358.153

Ippolito, M. ( 2004): "Imperfect Modality", in J. Guéron \& J. Lecarme (eds.): The Syntax of Time. Cambridge: MIT Press, pp. 359-387.

Izvorski, R. (1997): “The Present Perfect as an Epistemic Modal”, in A. Lawson (ed.)

Proceedings of SALT 7. Ithaca: CLC Publications, pp. 222-239.

http://dx.doi.org/10.3765/salt.v7i0.2795

KanY, C. (1970): Sintaxis hispanoamericana. Madrid: Gredos.

KLEIN, W. (1992): “The present perfect Puzzle”, in Language 68, pp. 525-552. http://dx.doi.org/10.2307/415793

KLeIN, W. (1994): Time in Language. Londres: Routledge.

Koev, T. (2011): "Evidentiality and temporal distance learning", in N. Ashton, A. Chereches \& D. Lutz (eds.) Proceedings of SALT 21. New Brunswick: Rutgers University, pp. 115-134. http://dx.doi.org/10.3765/salt.v21i0.2594

Kratzer, A. (1991): Modality, in D. Wunderlich \& A. von Stechow (eds.): Semantics: An International Handbook of Contemporary Research. Berlín: de Gruyter, pp. 639-650.

Kratzer, A. (2012): Modals and Conditionals, cap. 2. Oxford: Oxford University Press.

Krivoshein, N. \& F. Acosta (2007). Gramática guaraní. Asunción: Servilibro.

LeE, J. (2011): "The Korean evidential -te: A modal analysis", in O. Bonami \& P. Cabredo Hofherr (eds.): Empirical Issues in Syntax and Semantics 8, pp. 287-311.

Lefebvre, C. \& P. Muysken (1988): Mixed categories: Nominalizations in Quechua. Dordrecht: Kluwer Academic Publishers.

Martín, H. (1976): “Un caso de interferencia en el español paceño”, Filología 17-18, pp. 119-130.

Matthewson, L. (2011): “On apparently non-modal evidentials", in O. Bonami and P. Cabredo Hofherr (eds.): Empirical Issues in Syntax and Semantics 8, pp. 333-357. Matthewson, L., H. Davis \& H. Rullmann (2007): "Evidentials as epistemic modals: Evidence from St'át'imcets", Linguistic Variation Yearbook 7 (1), pp. 201-254. Melià, B. 1992. La lengua guarani del Paraguay. Madrid: Mapfre. 
Melià, B., L. FARré \& A. Pérez (1958): El guaraní a su alcance. Un método para aprender la lengua guaraní del Paraguay. Asunción: CEPAG.

Molochieva, Z. (2007): "Category of Evidentiality and Mirativity in Chechen". Ponencia presentada en el evento Conference on Languages of the Caucasus, Leipzig, 9-12 de diciembre.

Olbertz, H. (2009): "Mirativity and exclamatives in functional discourse grammar: evidence from Spanish", in E. Keizer \& G. Wanders (eds.): The London papers I. Web Papers in Functional Discourse Grammar, vol. 82. Amsterdam: Functional Grammar Foundation, pp. 66-82.

Ortiz MaYAns, A. (1973): Nuevo diccionario español-guaraníguaraní español (10 ed.). Buenos Aires: Librería Platero Editorial/Librería Comuneros.

Parsons, T. (1990): Events in the Semantics of English. Cambridge: MIT Press.

Penner, H. (2007): "Se habla. Es guaraní. No es guaraní. Es castellano. No es castellano. Es guaraní y castellano. No es guaraní ni castellano. ¿Qué es?”, Signos Lingüísticos 5, pp. 45-95.

Peterson, T. (2010): "Examining the Mirative and Nonliteral Uses of Evidentials", in T. Peterson \& U. Sauerland (eds.): Evidence from Evidentials (UBCWPL 28). Vancouver: UBCWPL, pp. 129-159.

Peterson, T. (2013): "Rethinking Mirativity: The Expression and Implication of Surprise". Ms., University of Toronto.

[RAE] Real Academia Española y Asociación de Academias de la Lengua Española. (2009): Nueva gramática de la lengua española. Madrid: Espasa.

Reichenbach, H. (1947): Elements of Symbolic Logic. Nueva York: Macmillan.

RetT, J. \& S. Murray (2013): "A semantic account of mirative evidentials", in T. Snider (ed.) Proceedings of SALT 23, pp. 453-472. http://dx.doi.org/10.3765/salt. v23i0.2687

Rivero, M. ${ }^{a}$ L. (2014): "Spanish inferential and mirative futures and conditionals: An evidential gradable modal proposal", Lingua 151, pp. 197-215. http://dx.doi. org/10.1016/j.lingua.2014.04.009

SÁNCHEZ, L. (2004): "Functional convergence in the tense, evidentiality and aspectual systems of Quechua Spanish bilinguals", Bilingualism: Language and Cognition 7/2, pp. 147-162. http://dx.doi.org/10.1017/S136672890400149X

SMIRnova, A. (2011): "The meaning of the Bulgarian evidential and why it cannot express inferences about the future", in N. Ashton, A. Chereches \& D. Lutz (eds.): Proceedings of SALT 21. New Brunswick: Rutgers University, pp. 275-297. http://dx.doi.org/10.3765/salt.v21i0.2618 
TAYLOR, J. (1984): “Marcação Temporal na Língua Kaiwá”, Série Linguística 11: pp. $37-121$.

TONHAUSER, J. (2006): The temporal semantics of noun phrases: Evidence from Guarani. Stanford: Stanford University, tesis doctoral.

Tonhauser, J. (2009): “Counterfactuality and Future Time Reference: The Case of Paraguayan Guaraní - mo 'â", in A. Riester \& T. Solstad: Proceedings of Sinn und Bedeutung 13. Stuttgart: Universität Stuttgart, pp. 527-541.

Tonhauser, J.(2011): "Temporal reference in Paraguayan Guaraní, a tenseless language", Linguistics and Philosophy 34, pp. 257-303. http://dx.doi.org/10.1007/ s10988-011-9097-2

Torres Bustamante, T. (2012): "Real past and real tense in mirativity", in A. Chereches (ed.) Proceedings of SALT 22, pp. 347-364.

Torres Bustamante, T. (2013): On the Syntax and Semantics of Mirativity: Evidence from Spanish and Albanian. New Brunswick: Rutgers University, tesis doctoral.

Vidal de Battini, B. (1980): Cuentos y leyendas populares de la Argentina. Buenos Aires: Ediciones culturales argentinas.

von Fintel, K. \& I. HeIm (2011): Intensional Semantics. MIT, ms.

WiLLET, T. (1988): “A cross-linguistic survey of the grammaticization of Evidentiality”, Studies in Language 12 (1), pp. 51-97. http://dx.doi.org/10.1075/s1.12.1.04wil 
